\title{
MACHINE COMPUTATION USING THE EXPONENTIALLY CONVERGENT MULTISCALE SPECTRAL GENERALIZED FINITE ELEMENT METHOD*
}

\author{
Ivo BABušKa ${ }^{1}$, Xu HuAng ${ }^{2}$ And Robert Lipton $^{3}$
}

\begin{abstract}
A multiscale spectral generalized finite element method (MS-GFEM) is presented for the solution of large two and three dimensional stress analysis problems inside heterogeneous media. It can be employed to solve problems too large to be solved directly with FE techniques and is designed for implementation on massively parallel machines. The method is multiscale in nature and uses an optimal family of spectrally defined local basis functions over a coarse grid. It is proved that the method has an exponential rate of convergence. To fix ideas we describe its implementation for a two dimensional plane strain problem inside a fiber reinforced composite. Here fibers are separated by a minimum distance however no special assumption on the fiber configuration such as periodicity or ergodicity is made. The implementation of MS-GFEM delivers the discrete solution operator using the same order of operations as the number of fibers inside the computational domain. This implementation is optimal in that the number of operations for solution is of the same order as the input data for the problem. The size of the MS-GFEM matrix used to represent the discrete inverse operator is controlled by the scale of the coarse grid and the convergence rate of the spectral basis and can be of order far less than the number of fibers. This strategy is general and can be applied to the solution of very large FE systems associated with the discrete solution of elliptic PDE.
\end{abstract}

Mathematics Subject Classification. 65N30, 74S05, 74Q05.

Received August 3, 2013.

Published online March 11, 2014.

\section{INTRODUCTION}

Over the past two hundred years there has been a sustained effort to develop quantitative theories for the analysis of multi-scale phenomena. The early investigations of Poission [46] (1822) sought to represent the physical response of heterogeneous media by an "equivalent" homogeneous one. The subsequent work of Mosotti (1850) [36], Maxwell (1873) [37], and Rayleigh (1892) [47] proposed methods for recovery of effective

\footnotetext{
Keywords and phrases. Generalized finite elements, multiscale method, spectral method, heterogeneous media, fiber reinforced composites.

* This work is supported by grants: NSF DMS-1211066 \& NSF DMS-1211014.

1 Institute for Computational Engineering and Science and Department of Aerospace Engineering, University of Texas, Austin, TX 78712, USA. babuska@ices.utexas.edu

2 Department of Mathematics, Louisiana State University, Baton Rouge, LA 70803, USA. xhuang4@tigers.1su.edu

3 Department of Mathematics and Center for Computation and Technology, Louisiana State University, Baton Rouge, LA 70803, USA. lipton@math.lsu.edu
} 
coefficients. It is interesting to note that this intense activity occurred a full century before the mathematical development of homogenization theory for partial differential equations in the 1960's. A survey of the nineteenth and early twentieth century literature on this topic is given in the 1926 review paper of Lichternecker [34]. New engineering challenges motivated by the technology of the mid 20th century brought research activity to bear on structural and transport properties of heterogeneous media including multiphase polymer systems [13], catalytic materials [52], and fiber reinforced composites [2, 18, 26, 28, 29]. The mathematical theory of homogenization embodied in the notion of G-convergence can be traced to the work of DeGiorgi ([49] example p. 661) and the work of Spagnolo $[49,50]$. More recent theory addresses problems with non-symmetric coefficients and the notion of H-convergence, [41]. Homogenization theory for periodic media and extensions to many important problems in mathematical physics are developed in the work of $[12,16,48]$. The modern mathematical theory of effective coefficients and their relation to microstructure has seen explosive development since the late 1980's and reviews of many important developments can be found in the recent monographs $[40,56]$.

Modern computational technology is driving the development of numerical approaches to multiscale problems. These approaches have gained traction in the applications and the last 40 years has witnessed a growing scientific literature addressing the numerical treatment of multiscale problems. The field has grown such that it is not possible to provide an exhaustive review within this paper. Here we refer to the recent monograph [21] for an extensive survey of this growing literature. Contemporary approaches to numerical multi-scaling can be split into two categories, exemplified by (1) Variational Multiscale Methods (VMS) and (2) Multiscale Finite Element Methods (MsFEM).

The scheme behind (VMS) [33] is to (a) additively decompose the solution space $V_{R}$ into fine and coarse scale contributions, (b) solve the fine scale equations as driven by the coarse scale residual, (c) use the fine scale solution operator $\mathcal{M}$ to eliminate the fine scale solution and represent it as a linear function of the coarse scale residual and (d) solve this modified problem over the coarse scale space. Here $\mathcal{M}$ is referred to as the reconstruction operator $[19,20]$ or as the fine scale Green's function [32]. The operator $\mathcal{M}$ is naturally related to the corrector appearing in homogenization theory [42]. In discrete implementations the space $V_{R}$ is the usual finite element (FE) space obtained on refining the grid associated with the FE coarse scale space $V_{C}$. Elements of the fine scale space $V_{F} \subset V_{R}$ are chosen to have compact support and taken to be orthogonal to $V_{C}$ in the $H_{0}^{1}$ inner product. To fix ideas the coarse scale space is spanned by hat functions associated with the coarse mesh. In this scheme it is seen that higher fidelity approximations to the nonlocal solution operator $\mathcal{M}$ are obtained by redefining $V_{F}$ to include functions with progressively larger support sets (oversampling). Adaptive oversampling methods within the frame work of VMS are introduced and developed in [35,42]. Recently novel approximation methods have been introduced that provide rigorous convergence rates for multiscale methods and are appropriate for the VMS scheme [15,43,44]. The use of harmonic coordinates [43] and the transfer property of the flux norm [15], and its localization [44] turn out to provide explicit rates of convergence for multi-scale methods of VMS type. For VMS posed over the coarse scale mesh of diameter $H$ a convergence rate of $\mathcal{O}(H)$ is seen to follow from suitable oversampeling [44]. The reader is also referred to the recent papers [35] for discussion of convergence rates for VMS and dependence on the support of the fine scale basis functions. Related but independent developments in multiscale approaches exploiting the interplay between local and global computation and homogenization theory are put forth in the Heterogeneous Multiscale Methods introduced in $[19,20,24]$.

The idea behind (MsFEM) is to consider a coarse mesh with dimensions larger than the heterogeneity of the medium and instead of using linear or polynomial $\mathrm{FE}$ basis functions one uses a finite dimensional space of local solutions to the problem over each element of the coarse mesh. This method has two components to it. The first is to create a local approximation space over each coarse element of the mesh and the second requires one to "paste" these elements together. This idea was suggested within the Partition of Unity Method (PUM) for pasting together local approximations and analyzed in one dimension in [10] and for higher dimensional problems using special local solutions in $[6]$ and generalized broadly see, $[9,21,38,54]$ and the citations given there. This idea but with a different strategy for pasting together local bases is proposed in $[22,23]$ and is analyzed in generality there. To illustrate the ideas developed in $[22,23]$ consider a problem in two dimensions and let $\omega$ be 
a triangular or quadrilateral element. Here have the following three basic choices for the construction of the local function space inside $\omega$.

1. Linear boundary data. We retain linearity at the boundary of the element $\partial \omega$ and generate local shape functions as solutions of the homogenous multiscale equation inside the element with boundary conditions on $\partial \omega$ associated with linear or bilinear FE. We point out there is a loss of accuracy in this approach since the trace of the exact solution over the boundary of the element is not well approximated by a linear function.

2. Better boundary approximation. To improve the boundary approximation over $\partial \omega$ we first solve a one dimensional problem on every edge of $\omega$ with boundary condition on the vertices taken to be the nodal values of FE functions. Typically the equation on each edge of the element is the restriction of the two dimensional problem to that edge. This approach leads to better accuracy and both approaches 1) and 2) deliver conforming elements.

3. Oversampling. Let $\omega^{*}$ be the triangle (quadrilateral) $\omega$ enlarged by a factor of 2 . Here we take $\omega \subset \omega^{*}$ to be concentric and the diameter of $\omega^{*}$ is twice that of $\omega$. We prescribe the traces of the linear FE shape functions on boundary of $\omega^{*}$. Using this boundary data we solve the local problem over $\omega^{*}$. The local approximation is then obtained on restricting these solutions to $\omega$. Here this approach leads to nonconforming elements. This approach to constructing local bases is referred to as oversampling [21].

Each approach listed above is a generalization of the linear FEM. It is evident that these methods immediately extend if one applies higher order FE boundary conditions on the edges and provides a corresponding higher order MsFEM. Several variations of these ideas have been applied and developed for multiscale problems and an overview of recent literature is given in [21].

We may also interpret MsFEM as a domain decomposition method. Here the elements $\omega$ appearing in the MsFEM could be understood as a domain decomposition of the computational domain $\Omega$, over which the problem is formulated. The methods 1) and 2) listed above are domain decompositions without overlap and can be understood as a simple mortar method. For further developments of the mortar approach to multiscale problems and significant generalizations along these lines see [1]. While the approach 3) employs oversampling and this delivers a domain decomposition with overlap.

We continue with the theme of domain decomposition and cover $\Omega$ with domains $\omega_{i}$ such that $\cup_{i} \omega_{i}=\Omega$. The multiscale approach has two components: 1) Local Approximation. On every $\omega_{i}$ we propose $m$ dimensional spaces $V_{i} \subset H^{1}\left(\omega_{i}\right)$ such that the exact solution $u$ for the multiscale problem can be well approximated by a function $v_{i} \in V_{i}$ over the element $\omega_{i}$ such that $\left.\left\|u-v_{i}\right\|_{\mathcal{E}\left(\omega_{i}\right)} \leq \varepsilon_{i} .2\right)$ Construction of a $H^{1}(\Omega)$ global approximation from local approximations. Here we suppose we have employed a scheme (e.g. partition of unity) for "pasting" the functions $v_{i}$ together to construct a "continuous" function $v$ belonging to $H^{1}(\Omega)$ and

$$
\sum_{i}\|u-v\|_{\mathcal{E}\left(\omega_{i}\right)}^{2} \leq C \sum \varepsilon_{i}^{2}
$$

where $\|\cdot\|_{\mathcal{E}\left(\omega_{i}\right)}$ is the energy norm over the element $\omega_{i}$ and $C$ independent of $u$ and $v_{i}$. As in the previous case one is faced with the dual issues of finding accurate local approximations and the global problem of combining the local approximations to obtain an approximation to the solution $u$. Here the local functions could be pasted or combined to form a global function using non-overlapping or overlapping elements.

The Generalized Finite Element Method (GFEM) is an overlapping domain decomposition method where global approximations are obtained by pasting together local approximations through a partition of unity. Partition Unity Methods (PUM) originated in [6] and were further extended and analyzed in $[4,5,9,38]$ and applied to multiscale problems in $[3,25,53-55]$. The GFEM utilizes the results of independent local computations carried out across the computational domain. The GFEM is constructed by covering the computational domain $\Omega$ by a collection of preselected subsets $\omega_{i}, i=1,2, \ldots, n$ and constructing finite dimensional approximation spaces $\Psi_{i}$ over each subset using local information. The the global approximation is constructed by pasting the local approximations together using the partition of unity functions subordinate to the covering $\left\{\omega_{i}\right\}_{i=1}^{n}$. Since each 
space $\Psi_{i}$ is computed independently the full "global" solution is obtained by solving a global (macro) system which is an order of magnitude smaller than the system corresponding to a direct application the finite element method to the full structure. This provides an opportunity for the significant reduction of the computational work involved in the numerical modeling of large heterogeneous problems. Several advantages for applying this strategy to compute fields inside heterogeneous media are listed below:

1. Solution of a global problem with drastically reduced degrees of freedom.

2. Independent local mesh generation versus the generation of a globally defined mesh.

3. Completely independent parallel computation of local problems.

Recent work [7] identifies optimal local finite dimensional approximation spaces $\Psi_{i}$ for rough $\left(L^{\infty}\right)$ coefficients. These new approximation spaces, related to the spectra of restriction operators, provide exponential accuracy in terms of the local degrees of freedom $\operatorname{dim}\left(\Psi_{i}\right)$. In principal this means that to achieve a global approximation error of $\tau$ with respect to the energy norm one needs to employ $\ln ^{d+1}\left(\frac{1}{\tau}\right)$ local basis functions on each subdomain. This newfound low dimensionality for special local approximation spaces expands the potential for high fidelity machine computation of elastic fields inside very large multiscale heterogeneous structures. The crucial aspect of our approach is that it allows for the solution of problems that are too large to solve using traditional FEM discretizations on a given computational resource. This type of approach developed here and in [7] is called Multiscale Spectral GFEM.

In related work local bases are defined for any shape regular mesh of size $H$ with $\mathcal{O}(H)$ convergence rate using $\ln ^{d+1} \frac{1}{H}$ bases functions per nodal point [27]. Here construction of the near field components of the local bases parallels the work of $[35,44]$ while the far field bases components are motivated by the approximants developed in the theory of $\mathcal{H}$-matrices [14]. The optimality of this type of local basis remains to be established.

In this paper we address the elastic problem and introduce the appropriate optimal local approximation spaces. As in [7] the best choice of local approximation is motivated by the Kolomoragov n-width and we use it to identify and prove the existence of spectrally defined finite dimensional optimal local approximation spaces. In doing so we establish the existence of an explicitly defined optimal local approximation space for this problem. We also provide an estimate to show that it is possible to achieve a local approximation error of $\tau$ with respect to the energy norm using at most $\ln ^{d+1}\left(\frac{1}{\tau}\right)$ local basis functions see, Section 7 . We point out that numerical experiments show that the approximation error can be actually be achieved using far less local basis functions in the pre-asymptotic regime see, [55]. With these estimates in hand the current paper addresses the numerical implementation of Multiscale Spectral GFEM (MS-GFEM) for a fiber reinforced composite medium. For this case we identify the relevant error estimate in Section 5 and estimates for machine time required for constructing an approximate solution within a prescribed tolerance $\tau$ using a parallel computer in 6 . Moreover the size of the MS-GFEM matrix used to represent the discrete inverse operator is small on the order of $\ln ^{2(d+1)}\left(\frac{1}{\tau}\right)$. Along the way we identify open problems in elliptic regularity theory and challenges facing numerical implementation on large parallel computers. We show how these issues influence our ability to carry out machine computation for very large multiscale problems as exemplified by the composite example treated here.

\section{THE FIBER COMPOSITE PROBLEM}

The over all methodology formulated in this paper is quite general and applies to mathematical formulations of linear elasticity described by measurable tensor valued coefficients. Such generality is required for the development of mathematically rigorous solution strategies. On the other hand the machine computation of displacement fields inside engineering materials requires a precise description of the heterogeneous material properties. We begin with the general formulation of the equilibrium problem for an anisotropic heterogeneous linearly elastic medium and then specialize our treatment to two dimensional plane strain problems for unidirectional fiber reinforced composites.

Let $\Omega \in \mathbb{R}^{d}, d=2,3$ be a bounded domain with Lipschitz boundary $\partial \Omega$. We start by formulating the problem for the system of linear elasticity used for the determination of elastic displacement fields $\mathbf{u}_{0}: \Omega \mapsto \mathbb{R}^{d}$. The 
equilibrium equation of linear elasticity is given by

$$
-\operatorname{div}\left(\mathbb{A}(\mathbf{x}) e\left(\mathbf{u}_{0}(\mathbf{x})\right)\right)=\mathbf{f}(\mathbf{x}), \quad \mathbf{x} \in \Omega .
$$

Where $e\left(\mathbf{u}_{0}\right)$ is the elastic strain and is the symmetric part of the gradient of the displacement $\nabla \mathbf{u}_{0}$ given by $e\left(\mathbf{u}_{0}\right)=\left(\nabla \mathbf{u}_{0}+\nabla \mathbf{u}_{0}^{T}\right) / 2$. The elasticity tensor of an anisotropic heterogeneous medium is characterized by a measurable tensor valued field $\mathbb{A}_{i j k l}(x) \in L^{\infty}(\Omega)$ for $i, j, k, l=1, d$. $\mathbb{A}_{i j k l}=\mathbb{A}_{i j l k}=\mathbb{A}_{j i k l}=\mathbb{A}_{k l i j}$. We suppose the tensor satisfies the standard coercivity and boundedness conditions and for any symmetric $d \times d$ matrix $e$

$$
\alpha|e|^{2} \leq \mathbb{A} e: e \leq \beta|e|^{2}, \mathbf{x} \in \Omega
$$

where $\mathbb{A} e: e=\mathbb{A}_{i j k l} e_{i j} e_{k l}$, and $|e|^{2}=e_{i j}^{2}$. For this choice of elasticity coefficient the solution is sought in the Sobolev space $H^{1}\left(\Omega ; \mathbb{R}^{d}\right)$ and the right-hand side (the body force) lies in the dual space $H^{1}\left(\Omega ; \mathbb{R}^{d}\right)^{*}$.

The mathematical formulation of our physical problem is a boundary value problem for the strongly elliptic system given by the linear elastic system (2.2). The weak solution is formulated in the standard variational way. We introduce the "energy" bilinear form

$$
B(\mathbf{u}, \mathbf{v})=\int_{\Omega} \mathbb{A} e(\mathbf{u}): e(\mathbf{v}) \mathrm{d} \mathbf{x}, \mathbf{u}, \mathbf{v} \in H^{1}\left(\Omega ; \mathbb{R}^{d}\right),
$$

and the energy norm

$$
\|\mathbf{u}\|_{\mathcal{E}(\Omega)}=(B(\mathbf{u}, \mathbf{u}))^{1 / 2} .
$$

By $\mathcal{E}(\Omega)$ we define the energy space given by the quotient space $H^{1}\left(\Omega ; \mathbb{R}^{d}\right) / \mathcal{R}$ equipped with the energy norm. Here the linear space of rigid motions $\mathcal{R}$ is given by

$$
\mathcal{R}=\left\{\mathbf{a}+\mathbf{b} \wedge \mathbf{x} ; \mathbf{a} \text { and } \mathbf{b}, \text { in } \mathbb{R}^{d}\right\} .
$$

In what follows we will write $B(\mathbf{u}, \mathbf{v})=(\mathbf{u}, \mathbf{v})_{\mathcal{E}(\Omega)}$.

Let $F_{1}(\mathbf{v})=\int_{\partial \Omega} \mathbf{v} \cdot \mathbf{g} \mathrm{d} \mathbf{x}$, be the functional of the tractions and $F_{2}(\mathbf{v})=\int_{\Omega} \mathbf{f} \cdot \mathbf{v} \mathrm{d} \mathbf{x}$, be the load functional. We assume that the natural consistency condition between $F_{1}$ and $F_{2}$ given by

$$
F_{1}(\mathbf{v})+F_{2}(\mathbf{v})=0, \text { for all } \mathbf{v} \in \mathcal{R}
$$

is satisfied.

The elastic displacement field $\mathbf{u}_{0}$ in $\Omega$ is the solution of the problem, $\mathbf{u}_{0} \in \mathcal{E}(\Omega)$,

$$
B\left(\mathbf{u}_{0}, v\right)=F(\mathbf{v})=F_{1}(\mathbf{v})+F_{2}(\mathbf{v}), \forall \mathbf{v} \in \mathcal{E}(\Omega),
$$

and is uniquely specified up to a rigid motion. If additionally on $\Gamma \subset \partial \Omega$ the boundary condition is $\mathbf{u}=\mathbf{u}_{\Gamma}$ then the unique solution $\mathbf{u}_{0} \in \mathcal{E}(\Omega), \mathbf{u}_{0}=\mathbf{u}_{\Gamma}$ satisfies $B\left(\mathbf{u}_{0}, \mathbf{v}\right)=F(\mathbf{v}), \forall \mathbf{v} \in \mathcal{E}(\Omega), \mathbf{v}=0$ on $\Gamma$. If $F_{2}=0$ then $\mathbf{u}_{0}$ is the $\mathbb{A}$-harmonic function satisfying $B\left(\mathbf{u}_{0}, \mathbf{v}\right)=0, \forall \mathbf{v} \in C_{0}^{\infty}$

The solution of a particular physical problem requires the specialization of the general formulation to the case at hand. Here we focus on the physical problem of calculating stresses and strains inside a uni-directional carbon fiber epoxy resin composite. This type of structural composite is commonly used in commercial aircraft and wind turbines primarily due to its high specific stiffness and strength. The principle objective of this paper is to describe the problem of numerical calculation of local fields inside engineering composite systems. The goal is to highlight the issues and problems related to machine computation of local fields inside structural composites typically requiring $10^{8}$ degrees of freedom per square centimeter of fibrous composite material.

We formulate a deterministic two dimensional elasticity problem for a uni-directional fiber reinforced composite. It is assumed that the fiber positions and diameters are available through image data. In the the two dimensional formulation we make the idealization and assume that the fiber cross sections are are circular. 


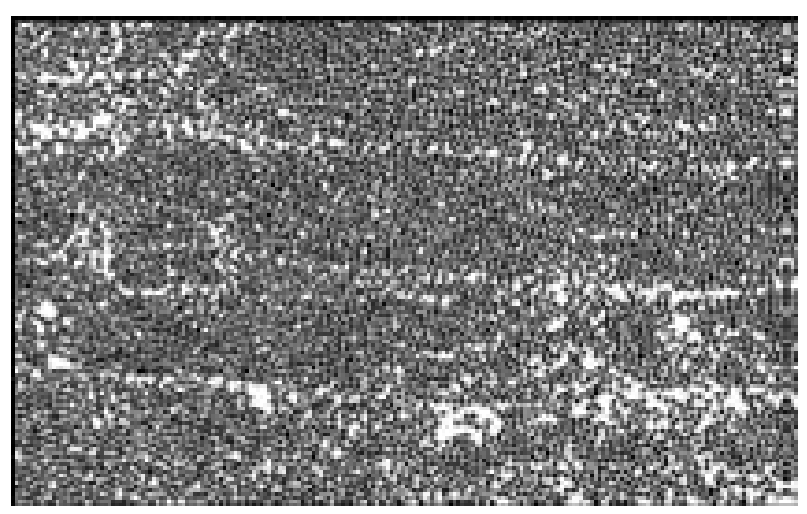

Figure 1. Fiber-reinforced composite.

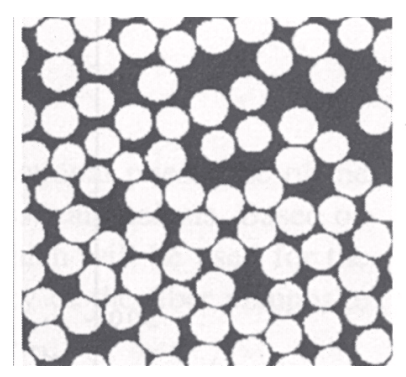

Figure 2. Microstructure.

In actuality the fibers are more like deformed cylinders. Here we emphasize that the fiber distribution is not periodic, so that the theory and computational analysis based on the assumption of a periodicity or almost periodicity characterized by an elastic tensor field of the type $\mathbb{A}(\mathbf{x}, \mathbf{x} / \varepsilon)$ where the tensor field $\mathbb{A}$ is smooth in the first variable periodic and oscillatory in the second variable cannot be used.

We will consider the square domain $\Omega=(-\kappa, \kappa) \times(-\kappa, \kappa)$ where the length $\kappa$ is given in meters denoted by $\mathrm{m}$. The boundary of $\Omega$ is denoted $\partial \Omega$. We have $|\Omega|=4 \kappa^{2}[\mathrm{~m}]^{2}$. We assume that the fiber volume fraction is $\mathrm{W} \simeq 50 \%$. The fiber cross sections are circular with diameters $\bar{d}$ ranging between $5 \mu \mathrm{m} \leq \bar{d} \leq 10 \mu \mathrm{m}$ Here $1 \mu \mathrm{m}=10^{-6} \mathrm{~m}$. The fibers provide structural stiffness and their stiffness greatly exceeds the matrix. The domain $\Omega$ contains approximately $\left(2 \times 10^{5} \kappa\right)^{2}$ fibers. As indicated we assume that the locations and the diameters of the fibers are known from image data see, Figures 1, 2 and for more see, [3].

For this case the elasticity tensor field is piecewise constant and of the form

$$
\mathbb{A}_{i j k l}(\mathbf{x})=\frac{E(\mathbf{x})}{2(1-\nu(\mathbf{x}))} \delta_{i j} \delta_{k l}+\frac{E(\mathbf{x})}{2(1+\nu(\mathbf{x}))}\left\{\delta_{i l} \delta_{j l}+\delta_{i l} \delta_{j k}-\delta_{i j} \delta_{k l}\right\}
$$

where

$$
\begin{aligned}
& E(\mathbf{x})=24 \mathrm{GPa} \text { for } \mathbf{x} \text { in the fibers, } 3.6 \mathrm{GPa} \text { for } \mathbf{x} \text { in the epoxy matrix } \\
& \nu(\mathbf{x})=0.24 \text { for } \mathbf{x} \text { in the fibers, } 0.3 \text { for } \mathbf{x} \text { in the epoxy matrix. }
\end{aligned}
$$

The mathematical formulation of the two dimensional plane strain problem is given by (2.8) with $\mathbb{A}(\mathbf{x})$ given by (2.9) and boundary conditions given by traction in units of GPa or displacement in units of meters m. Here $\mathbf{x}=\left(x_{1}, x_{2}\right), \mathbf{u}=\left(u_{1}, u_{2}\right)$, where both quantities are expressed in units of meters $\mathrm{m}$. The elasticity tensor field 
$\mathbb{A}(\mathbf{x})$ defines the isotropic elastic properties of the matrix and the fibers. It is evident that the tensor $\mathbb{A}(\mathbf{x})$ is varies rapidly across the structure and hence is very "rough" see, Figures 1 and 2. The assumption that the fibers are represented by cylinders is an idealization. However it serves to illustrate the ideas and challenges behind machine computation of fields inside complex media.

The basic goal of this paper is to formulate and develop a multi-scale and highly parallelizable numerical method for obtaining approximate solutions $\mathbf{u}_{0, \text { appr }}$ to elastic deformations inside composite structures that satisfy $\left\|\mathbf{u}_{0}-\mathbf{u}_{0, \text { appr }}\right\|_{\mathcal{E}(\Omega)} \leq \tau\left\|\mathbf{u}_{0}\right\|_{\mathcal{E}(\Omega)}$ were $\tau$ is a given tolerance.

\section{Generalized finite Elements And MUltiscale formulation}

For completeness we now review the basic theory of the GFEM method as it applies to the problem treated here. For the proofs we refer to [4]. The elastic deformation $\mathbf{u}_{0}$ inside the composite is a solution of the problem $\mathbf{u}_{0} \in \mathcal{E}(\Omega), \mathbf{u}_{0}=\mathbf{u}_{\Gamma}$ on $\Gamma \subset \partial \Omega$,

$$
B\left(\mathbf{u}_{0}, \mathbf{v}\right)=F(\mathbf{v}), \forall \mathbf{v} \in \mathcal{E}(\Omega), \mathbf{v}=\mathbf{0} \text { on } \Gamma
$$

Let $\left\{\Omega_{j}\right\}_{j=1}^{N}, j=1,2 \ldots, N$ be open set of open patches satisfying $\Omega_{j} \subset \Omega$ and $\Omega=\cup_{j=1}^{N} \omega_{j}$ and assume that any $\mathbf{x} \in \Omega$ belongs at most to $\varsigma$ patches $\omega_{j}$. Let $\left\{\phi_{j}\right\}_{j=1}^{N}$ be family of functions defined on $\Omega$, having piecewise continuous derivatives, and satisfying the following properties

$$
\begin{gathered}
\phi_{j}(\mathbf{x})=0, \text { for } \mathbf{x} \in \Omega \backslash \omega_{j}, j=1,2, \ldots, N, \\
\sum_{j=1}^{N} \phi_{j}(\mathbf{x})=1, \forall \mathbf{x} \in \Omega, \\
\max _{\mathbf{x} \in \Omega}\left|\phi_{j}(\mathbf{x})\right| \leq C_{1}, j=1,2, \ldots, N \\
\max _{\mathbf{x} \in \Omega}\left|\nabla \phi_{j}(\mathbf{x})\right| \leq C_{2} / \operatorname{diam}\left(\omega_{j}\right), j=1,2, \ldots, N
\end{gathered}
$$

where $0 \leq C_{1}, C_{2}<\infty$ and $\operatorname{diam}\left(\omega_{j}\right)$ is diameter of $\omega_{j}$. It is evident that $\left\{\phi_{j}\right\}$ is a partition of unity on $\Omega$. To every $\omega_{j}$ we associate on $\omega_{j}$ an $m(j)+3$-dimensional space $V_{j}$ given by the span of functions $\xi_{j i}=\left({ }^{1} \xi_{j i}\right.$, $\left.{ }^{2} \xi_{j i}\right) \in \mathcal{E}(\Omega), i=1,2, \ldots, m(j)$, together with the span of the basis, $\xi_{j, m(j)+1}=(1,0), \xi_{j, m(j)+2}=(0,1)$, $\xi_{j, m(j)+3}=\left(-x_{2}, x_{1}\right)$ for the three dimensional space of rigid motions in $\mathbb{R}^{2}$ denoted by $\mathcal{R}$. Let

$$
\xi_{j}=\sum_{i=1}^{m(j)+3} b_{j i} \xi_{j i}, \quad b_{j i} \in \mathbb{R}, \quad i=1, \ldots, m(j)+3 .
$$

Let $S$ be the span of all functions of the form

$$
\psi=\sum_{j=1}^{N} \phi_{j} \xi_{j}, \quad \xi_{j} \in V_{j}
$$

or equivalently $S$ is given by the span

$$
S=\operatorname{span}\left\{\eta_{j i}, \quad i=1,1,2, \ldots, m(j)+3, \quad j=1,2, \ldots, N\right\} .
$$

where

$$
\eta_{j i}=\phi_{j} \xi_{j i} \in \mathcal{E}(\Omega) .
$$

The space $V_{j}$ is called a local approximation space and the space $S$ the finite element space. Now we have Theorem 3.1. 
Theorem 3.1. Suppose first that $\Gamma=\emptyset$, i.e, only boundary tractions on $\partial \Omega$ tractions are prescribed. 1 . Every $V_{j}$ contains the subspace of rigid body motions and $\|\mathbf{v}\|_{L_{2}\left(\omega_{j}\right)} \leq C_{3} \operatorname{diam}\left(\omega_{j}\right)\|\mathbf{v}\|_{\mathcal{E}\left(\omega_{j}\right)}$ for all $\mathbf{v} \in \mathcal{E}\left(\omega_{j}\right)$ satisfying $\int_{\omega_{j}}(\mathbf{v} \cdot \mathbf{r}) d \omega_{j}=0$ where $\mathbf{r}$ is a rigid body motion. 2. Let $\mathbf{u} \in \mathcal{E}(\Omega)$ and for every $j$ there is $\xi_{j} \in V_{j}$ such that

$$
\left\|\mathbf{u}-\xi_{j}\right\|_{\mathcal{E}\left(\omega_{j}\right)} \leq \epsilon_{j}
$$

then there exists $\bar{\xi}_{j} \in V_{j}$ such that for

$$
\xi=\sum_{j=0}^{N} \phi_{j} \bar{\xi}_{j}
$$

we have

$$
\|\mathbf{u}-\xi\|_{\mathcal{E}(\Omega)} \leq C_{4}\left(\sum_{j=1}^{N} \epsilon_{j}^{2}\right)=C_{4} \epsilon
$$

with $C_{4}=\left(2 \varsigma\left(C_{1}^{2}+C_{2}^{2} C_{3}^{2}\right)\right)^{1 / 2}$. If $\Gamma \neq \emptyset$ and $\bar{\omega}_{j} \cap \Gamma$, then $V_{j}$ is the hyperplane such that if $\xi \in V_{j}$ then $\xi=\mathbf{u}_{\Gamma}$ on $\bar{\omega}_{j} \cap \Gamma$

Now we are able to formulate the GFEM. Consider the boundary value problem (3.11) and assume first that $\Gamma=\emptyset$. Let $\mathbf{u}_{0}$ be the solution of the problem (3.11), i.e., $\mathbf{u}_{0} \in \mathcal{E}(\Omega)$ satisfies

$$
B\left(\mathbf{u}_{0}, \mathbf{v}\right)=F(\mathbf{v}), \forall \mathbf{v} \in \mathcal{E}(\Omega) .
$$

and the solution is unique up to rigid body motion. Now the GFEM approximation $\mathbf{u}_{0 \text {,appr }} \in S$, satisfies

$$
B\left(\mathbf{u}_{0, \text { appr }}, \mathbf{v}\right)=F(\mathbf{v}), \mathbf{v} \in S .
$$

and the solution is also unique up to rigid body motion. It immediately follows from Theorem 3.1 that

$$
\left\|\mathbf{u}_{0}-\mathbf{u}_{0, \text { appr }}\right\|_{\mathcal{E}(\Omega)} \leq C_{4} \epsilon
$$

If $\Gamma \neq \emptyset$, and $\bar{\omega}_{j} \cap \Gamma \neq 0$ we can assume that once the space $V_{j}$ is properly modified that (3.25) holds.

\section{Optimal Local approximation spaces VIA SPECTRAL BASES}

In this section we introduce the optimal local bases. The basis functions appear as solutions of a special kind of eigenvalue problem involving $\mathbb{A}$ harmonic functions. In fact it turns out that it is impossible to improve on their approximation properties; this is shown in Section 7 where an exponential upper bound on the local approximation error is developed.

Let $\omega \subset \omega^{*} \subset \Omega \subset \mathbb{R}^{2}$ be two concentric square domains inside the composite material introduced in Section 3. We choose an intermediate length scale $H$. We assume $H$ is larger than the length scale of the fiber diameter and fiber spacing $\bar{d}$ but smaller than the dimensions of the domain $\Omega$ containing the composite. To fix ideas we write $H=\gamma \bar{d}$, where $\gamma \sim 1 \times 10^{2}$. Here we take $\omega=(-(1+\alpha) H / 2,(1+\alpha) H / 2)^{2}, 0<\alpha<1$ and $\omega^{*}=(-(1+\alpha) H,(1+\alpha) H)^{2}$. The energy inner products associated with these subsets are defined by

$$
\begin{aligned}
(\mathbf{u}, \mathbf{v})_{\mathcal{E}\left(\omega^{*}\right)} & =\int_{\omega^{*}} \mathbb{A} e(\mathbf{u}): e(\mathbf{v}) \mathrm{d} \mathbf{x} . \\
(\mathbf{u}, \mathbf{v})_{\mathcal{E}(\omega)} & =\int_{\omega} \mathbb{A} e(\mathbf{u}): e(\mathbf{v}) \mathrm{d} \mathbf{x} .
\end{aligned}
$$


For any open subset $S$ of the computational domain $\Omega$ we introduce the space of functions $H_{\mathbb{A}}\left(S ; \mathbb{R}^{2}\right)$ defined to be the functions in $H^{1}\left(S ; \mathbb{R}^{2}\right)$ that are $\mathbb{A}$-harmonic on $S$, i.e., $\mathbf{v} \in H^{1}\left(S ; \mathbb{R}^{2}\right)$ and

$$
(\mathbf{v}, \varphi)_{\mathcal{E}(S)}=\int_{S} \mathbb{A} e(\mathbf{v}): e(\varphi) \mathrm{d} x, \quad \forall \varphi \in C_{0}^{\infty}\left(S ; \mathbb{R}^{d}\right) .
$$

Since we work with the energy inner product we introduce the quotient space of $H_{\mathbb{A}}\left(\omega^{*} ; \mathbb{R}^{2}\right)$ with respect to rigid motions $\mathcal{R}$ denoted by $H_{\mathbb{A}}\left(\omega^{*} ; \mathbb{R}^{2}\right) / \mathcal{R}$. We also introduce the subspace $H_{\mathbb{A}}^{0}\left(\omega^{*} ; \mathbb{R}^{2}\right)$ given by elements of $H_{\mathbb{A}}\left(\omega^{*} ; \mathbb{R}^{2}\right)$ perpendicular to rigid motions with respect to the $L^{2}\left(\omega^{*} ; \mathbb{R}^{2}\right)$ inner product. Here we recall that $H_{\mathbb{A}}\left(\omega^{*} ; \mathbb{R}^{2}\right) / \mathcal{R}$ equipped with the energy norm is isometric to $H_{\mathbb{A}}^{0}\left(\omega^{*} ; \mathbb{R}^{2}\right)$. We define the linear map $\mathcal{T}$ from $H_{\mathbb{A}}\left(\omega^{*} ; \mathbb{R}^{2}\right) / \mathcal{R}$ into $H_{\mathbb{A}}\left(\omega ; \mathbb{R}^{2}\right) / \mathcal{R}$ as the restriction of $\mathbf{u} \in H_{\mathbb{A}}\left(\omega^{*} ; \mathbb{R}^{2}\right) / \mathcal{R}$ to $\omega$, i.e., $\mathcal{T} \mathbf{u}(x)=\mathbf{u}(x)$ for $\mathbf{x} \in \omega$. The operator $\mathcal{T}$ is compact, this follows immediately from an application of the the Caccioppoli inequality Lemma 7.6 together with the Rellich Kondrachov embedding theorem on $\omega^{*}$ see [7]. Let $\mathcal{T}^{*}$ be the adjoint mapping i.e., $(\mathcal{T} \varphi, \psi)_{\mathcal{E}(\omega)}=\left(\varphi, \mathcal{T}^{*} \psi\right)_{\mathcal{E}\left(\omega^{*}\right)}$ for all $\varphi \in H_{\mathbb{A}}\left(\omega^{*} ; \mathbb{R}^{2}\right) / \mathcal{R}$ and $\psi \in H_{\mathbb{A}}\left(\omega ; \mathbb{R}^{2}\right) / \mathcal{R}$ and the operator $\mathcal{T}^{*} \mathcal{T}$ is a compact operator mapping $H_{\mathbb{A}}\left(\omega^{*} ; \mathbb{R}^{2}\right) / \mathcal{R}$ into itself. The eigenvalue problem for the self adjoint bounded compact operator is written

$$
\mathcal{T}^{*} \mathcal{T} \varphi=\lambda \varphi .
$$

The eigenfunction, eigenvalue pairs are denoted by $\varphi_{j}$ and $\lambda_{j}, j=1,2, \ldots$ With $\lambda_{j}>0$ and the eigenvalues form a decreasing sequence $\lambda_{j} \geq \lambda_{j+1}$. The eigenvalues $\lambda_{j} \leq 1, j=1,2, \ldots$ are shown to decay exponentially i.e.,

$$
\lambda_{j} \simeq \mathrm{e}^{-j^{q}},
$$

where $q=\frac{1}{3}-\epsilon$. For three dimensional problems $q=\frac{1}{4}-\epsilon$, see Theorem 7.3, equation (7.58) and the discussion in Section 7. The eigenfunctions $\varphi_{j}$ form a complete orthonormal system for $H_{\mathbb{A}}\left(\omega^{*} ; \mathbb{R}^{2}\right) / \mathcal{R}$, i.e., $\left(\varphi_{i}, \varphi_{j}\right)_{\mathcal{E}\left(\omega^{*}\right)}=0$ for $i \neq j,\left\|\varphi_{j}\right\|_{\mathcal{E}\left(\omega^{*}\right)}=1$. In addition they enjoy orthogonality on $\omega$, i.e., $\left(\varphi_{i}, \varphi_{j}\right)_{\mathcal{E}(\omega)}=0$ for $i \neq j$ and

$$
\left\|\varphi_{j}\right\|_{\mathcal{E}(\omega)}=\lambda_{j}^{1 / 2}\left\|\varphi_{j}\right\|_{\mathcal{E}\left(\omega^{*}\right)} .
$$

For $\varphi \in H_{\mathbb{A}}\left(\omega^{*} ; \mathbb{R}^{2}\right) / \mathcal{R}$ one has

$$
\begin{aligned}
& \varphi=\sum_{j=1}^{\infty} c_{j} \varphi_{j}, \text { with } c_{j}=\left(\varphi, \varphi_{j}\right)_{\mathcal{E}\left(\omega^{*}\right)} \\
& \sum_{j=1}^{\infty} c_{j}^{2}=\|\varphi\|_{\mathcal{E}\left(\omega^{*}\right)}^{2} \text { and }\|\varphi\|_{\mathcal{E}(\omega)}^{2}=\sum_{j=1}^{\infty} c_{j}^{2} \lambda_{j} .
\end{aligned}
$$

From which we deduce the approximation property

$$
\left\|\varphi-\sum_{j=1}^{m} c_{j} \varphi_{j}\right\|_{\mathcal{E}(\omega)}^{2}=\sum_{j=m+1}^{\infty} c_{j}^{2} \lambda_{j} \leq \lambda_{m+1}\|\varphi\|_{\mathcal{E}\left(\omega^{*}\right)}^{2} .
$$

It now follows that any $\mathbb{A}$ harmonic function $\varphi \in H_{\mathbb{A}}\left(\omega^{*} ; \mathbb{R}^{2}\right) / \mathcal{R}$ can be approximated over $\omega$ by the $m$ dimensional space $V=\operatorname{span}\left\{\varphi_{j}: j=1,2, \ldots, m\right\}$ with the error given by $\lambda_{m+1}\|\varphi\|_{\mathcal{E}\left(\omega^{*}\right)}$. Moreover this choice of space $V$ is optimal in the sense that no other other $m$ dimensional space can lead to a better approximation for all $\varphi \in H_{\mathbb{A}}\left(\omega^{*} ; \mathbb{R}^{2}\right) / \mathcal{R}$. This is shown in Section 7 . It is pointed out that the optimal approximation space is not necessarily unique.

Presently the a priori knowledge of the higher regularity of the eigenfunctions $\left\{\varphi_{j}\right\}$ for fiber composites remains an open question. As of now it is not known if the eigenfunctions belong to the Besov space $H^{\alpha}\left(\omega^{*}\right)$, for some $\alpha>1$. With this in mind we make the hypothesis. 
Hypothesis 4.1. We consider the Besov space $B_{2, \infty}^{\alpha}\left(\omega^{*}\right)$ denoted by $B^{\alpha}\left(\omega^{*}\right)$ and suppose that the following inequality holds

$$
\left\|\varphi_{j}\right\|_{B^{\alpha}\left(\omega^{*}\right)} \leq C H^{-1 / 2} j^{\beta}\left\|\varphi_{j}\right\|_{\mathcal{E}\left(\omega^{*}\right)},
$$

with $\alpha=3 / 2$ and $\beta \geq 1 / 2$.

We assume $\alpha=3 / 2$ since the matrix $\mathbb{A}$ associated with the fiber reinforced composite in (2.4) is piecewise constant see, Figure 2. Here we will also assume that the fibers are not touching.

Remark 4.2. Here the exponent $\beta$ is not chosen. However because of the exponential approximation properties the maximum number of spectral basis elements required for a prescribed approximation error $\tau$ grows slowly, i.e., $j \leq\left[\ln \left(\tau^{-1}\right)\right]^{d+1}$. This feature mollifies the influence of the exponent $\beta$.

As before $\bar{d}$ denotes the length scale associated with the fiber diameters and distance between fibers. We consider a uniform mesh of diameter $h<\bar{d}$ chosen to deliver an approximation with an accuracy $\tau$. For this mesh the best piecewise bilinear finite element approximation to $\varphi_{j}$ is denoted by $\varphi_{j, h}^{*}$ and

$$
\left\|\varphi_{j}-\varphi_{j, h}^{*}\right\|_{\mathcal{E}\left(\omega^{*}\right)} \leq C h^{1 / 2}\left\|\varphi_{j}\right\|_{B^{3 / 2}\left(\omega^{*}\right)} .
$$

Combing this estimate with the regularity hypothesis (4.34) delivers the desired estimate in terms of the energy $\|\varphi\|_{\mathcal{E}\left(\omega^{*}\right)}$ given by

$$
\left\|\varphi_{j}-\varphi_{j, h}^{*}\right\|_{\mathcal{E}\left(\omega^{*}\right)} \leq C h^{1 / 2} H^{-1 / 2} j^{\beta}\|\varphi\|_{\mathcal{E}\left(\omega^{*}\right)} .
$$

Here we have addressed the approximation of $\mathbb{A}$ harmonic functions over local domains $\omega \subset \omega^{*}$. When the equilibrium equation $(2.2)$ is inhomogeneous on $\omega^{*}$ we can decompose the solution into $\mathbf{u}_{0}=\mathbf{u}-\chi+\chi$, where $\chi$ belongs to $H_{0}^{1}\left(\omega^{*} ; \mathbb{R}^{2}\right)$ and is the local particular solution of

$$
-\operatorname{div}(\mathbb{A}(\mathbf{x}) e(\chi(\mathbf{x})))=\mathbf{f}(\mathbf{x})
$$

for $\mathbf{x} \in \omega^{*}$. Here the difference $\mathbf{u}_{0}-\chi$ belongs to $H_{\mathbb{A}}\left(\omega^{*} ; \mathbb{R}^{2}\right)$ and can be approximated exponentially well using the spectral basis $\left\{\varphi_{j}\right\}_{j=1}^{m}$.

We now consider local domains $\omega$ that border the boundary $\partial \Omega$. The configuration of $\omega$ and $\omega^{*}$ for domains bordering the boundary is shown in Figure 3 see, section 7 . Here $\omega \subset \omega^{*}$ and both domains have boundary components that coincide with $\partial \Omega$. Spectral bases can be found for domains $\omega^{*}$ bordering the boundary $\partial \Omega$ for which the solution $\mathbf{u}_{0}$ is $\mathbb{A}$-harmonic and satisfies a homogeneous traction or Dirichlet condition on $\partial \omega^{*} \cup \partial \Omega$. The spectral bases for domains bordering the boundary also satisfy (4.31), (4.32), (4.33), and the associated eigenvalues decay exponentially (4.30) see, Theorem 7.5. When faced with non-homogeneous boundary conditions on $\partial \omega^{*} \cup \partial \Omega$, and body force $\mathbf{f}$ we solve for the local particular solution $\eta$ of

$$
-\operatorname{div}(\mathbb{A}(\mathbf{x}) e(\eta(\mathbf{x})))=\mathbf{f}
$$

for $\mathbf{x} \in \omega^{*}$ with non-homogeneous traction or Dirichlet boundary conditions on $\partial \omega^{*} \cup \partial \Omega$ and $\eta=0$ on $\partial \omega^{*} \cap \Omega$. For this case the difference $\mathbf{u}_{0}-\eta$ is $\mathbb{A}$-harmonic with homogeneous boundary conditions on $\partial \omega^{*} \cup \partial \Omega$ and can be approximated exponentially well by the spectral basis.

\section{Multiscale spectral GFEM and error estimates}

In this section we provide a priori error estimates for the discrete approximation of the composite material problem introduced in Section 2. We start by assuming that the local spectral bases can be computed with infinite accuracy. We apply this hypothesis and use the local spectral basis within the GFEM Galerkin scheme 


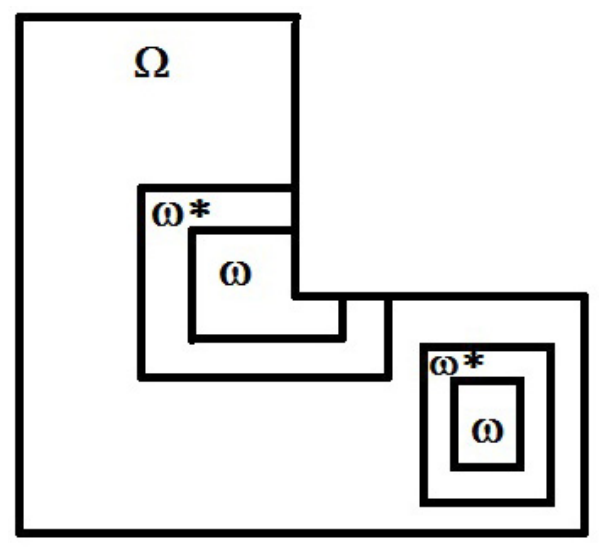

Figure 3. Local domains.

to provide the a priori error estimate given by (5.39). In the second part of this section we introduce the discrete finite element approximation to the local spectral bases. This is used together with (5.39) to establish the $a$ priori error estimate for the GFEM implementation using the discrete local approximation. This estimate is given by (5.48). This estimate allows us to assess the computational complexity of the multi-scale GFEM using optimal local bases provided in Section 6 .

Recall that the domain containing composite material is given by $\Omega=(-\kappa, \kappa)^{2}$. We cover it with a mesh defined by the nodal points $\mathbf{z}_{i, j}=\left(x_{i}, y_{j}\right), x_{i}=i H, y_{j}=j H$, with $i, j= \pm 0,1,2, \ldots, n$, and $H=\kappa /(1+\alpha)(n+$ $1 / 2)$ where $0<\alpha<1$ is chosen below. Let $Q_{i, j}$ be squares with center $\mathbf{z}_{i, j}$ and side length $\mu\left(Q_{i, j}\right)=H$. We define $\omega_{i, j}$ to be given by the square $Q_{i, j}^{\alpha}$ with center in $\mathbf{z}_{i, j}$ and side length $\mu\left(Q_{i, j}^{\alpha}\right)=(1+\alpha) H, 0<\alpha<1$. Further let $\omega_{i, j}^{*}=\Omega \cap Q_{i, j}^{\alpha^{*}}$ with $\alpha^{*}=\beta(1+\alpha)$. Now we fix $\alpha=1 / 4$ and $\beta=2$. For this choice the domains $\omega_{i j}^{*}$ are squares of side length $H^{*}=2.5 \mathrm{H}$. It is evident that $\Omega=\cup_{i, j} \omega_{i, j}$ and the domains $\omega_{i, j}$ overlap where the width of the overlap region is $H / 4$. In what follows domains $\omega_{i, j}$ bordering $\partial \Omega$, (i.e., $\omega_{i, j} \cap \partial \Omega \neq 0$ ) are called boundary subdomains and all others are referred to as interior subdomains. Consider interior subdomains $\omega_{i j}$ and let $\phi^{i, j}=1$ on $Q_{i, j}, \phi^{i, j}=0$ on $\Omega-\omega_{i, j}$ and be piecewise linear on $\omega_{i, j}-Q_{i, j}$. For boundary domains $\omega_{i, j}$ set $\phi^{i, j}=1$ over parts where $\omega_{i, j}$ does not overlap any other $\omega_{k, l}$. It is clear that these functions $\phi^{i, j}$ constitute a partition of unity i.e., $\sum_{i, j} \phi^{i, j}=1$ and can be employed within the GFEM see, Section 3.

On every interior domain $\omega_{i j} \subset \omega_{i, j}^{*} \subset \Omega$ the functions $\varphi_{i, j}^{(l)} l=1, \ldots, m(i, j)$ are elements the local spectral bases introduced in the previous section. Consider the span of the local basis $\varphi_{i, j}^{(l)}, l=1, \ldots, m(i, j)$ defined on $\omega_{i, j}^{*}$ together with the three dimensional span of the basis for rigid rotations in $\mathbb{R}^{2}$ and denote this $m(i, j)+3$ dimensional space by $W_{i, j}^{m(i, j)}$. If $\omega_{i, j}^{*}$ is a boundary subdomain and a non-homogenous boundary condition is prescribed on the boundary component common to $\partial \omega_{i, j}^{*}$ and $\partial \Omega$, then the space $W_{i, j}^{m(i, j)}$ is augmented by $\psi_{i, j}^{p}$ where the function $\psi_{i, j}^{p}$ is the $\mathbb{A}$-harmonic function satisfying the non-homogeneous boundary condition and $\psi_{i j}^{p}=0$ on $\partial \omega_{i j}^{*} \cap \Omega$. In addition if the right hand side is not zero the we augment the space $W_{i, j}^{m(i, j)}$ by a function $\chi_{i j}$ where $\chi_{i j}$ is a particular solution on of $\omega_{i j}^{*}$ with homogeneous Dirichlet boundary data on $\partial \omega_{i j}^{*}$. For the two dimensional problem treated here we have $N=(2 n)^{2}$ subdomains $\omega_{i j}$ and the associated partition of unity $\sum_{i j} \phi_{i j}=1$. We set $m=\min _{i j}\{m(i, j)\}$ and for this explicit application the multi-scale GFEM basis $W^{m}$ is given by the span of all functions of the form

$$
\varphi=\sum_{|i| \leq 1,|j| \leq n} \phi_{i j} \xi_{i j}, \xi_{i j} \in W_{i, j}^{m(i, j)}
$$


We write this as $W^{m}=\sum_{i, j} \phi_{i j} W_{i, j}^{m(i, j)}$ and $W^{m} \subset \mathcal{E}(\Omega)$. Let $\varphi^{p}=\sum_{i j} \phi_{i j} \psi_{i j}^{p}$ be the sum of local particular functions and set $\varphi=\sum_{i j} \phi_{i j} \xi_{i j}$ where $\xi_{i j}$ belong to the local spectral basis over $\omega_{i j}$. Then $\varphi^{p}+\varphi \in W^{m}$. Given a solution $\mathbf{u}_{0}$ of the boundary value problem (3.11) we consider its restriction to $\omega_{i j}$. Note that $\mathbf{u}_{0}-\left(\varphi^{p}+\varphi\right)=$ $\left(\mathbf{u}_{0}-\varphi^{p}\right)-\xi_{i j}$ on $\omega_{i j}$ and $\mathbf{u}_{0}-\varphi^{p}$ is $\mathbb{A}$-harmonic on $\omega_{i j}^{*}$ and we recall the exponential approximation property of the local spectral bases described in the previous section to see that we can construct a $\varphi \in W^{m}$ for which

$$
\begin{aligned}
\left\|\mathbf{u}_{0}-\left(\varphi^{p}+\varphi\right)\right\|_{\mathcal{E}\left(\omega_{i j}\right)} & =\left\|\left(\mathbf{u}_{0}-\varphi^{p}\right)-\varphi\right\|_{\mathcal{E}\left(\omega_{i j}\right)} \\
& \leq C_{0} \mathrm{e}^{-(m(i, j)+1)^{q}}\left\|\mathbf{u}_{0}-\varphi^{p}\right\|_{\mathcal{E}\left(\omega_{i j}^{*}\right)} \\
& \leq C_{1} \mathrm{e}^{-(m+1)^{q}}\left\|\mathbf{u}_{0}\right\|_{\mathcal{E}\left(\omega_{i j}^{*}\right)}
\end{aligned}
$$

Now we apply Theorem 3.1 to discover that there is an approximation $\varphi \in W^{m}$ for which

$$
\left\|\mathbf{u}_{0}-\varphi\right\|_{\mathcal{E}(\Omega)}^{2} \leq C \sum_{i, j} \mathrm{e}^{-2(m(i, j)+1)^{q}}\left\|\mathbf{u}_{0}\right\|_{\mathcal{E}\left(\omega_{i, j}^{*}\right)}^{2} \leq C^{2} \mathrm{e}^{-2(m+1)^{q}}\left\|\mathbf{u}_{0}\right\|_{\mathcal{E}(\Omega)}^{2}=C^{2} \tau^{2}\left\|\mathbf{u}_{0}\right\|_{\mathcal{E}(\Omega)}^{2}
$$

Here we have set $\tau=\mathrm{e}^{-(m+1)^{q}}$, where $q=\frac{1}{3}-\epsilon$. This is the a priori estimate for the error assuming a perfect computation of the local spectral bases. Here we view the local domains $\omega_{i j}$ as coarse elements of size $H$. In the estimate (5.39) we have assumed that the eigenvalue problem can be solved exactly.

With the estimate (5.39) in hand we now carry out the a priori estimate assuming a finite element approximation of the spectral bases on $\omega_{i j}^{*}$. Let $\mathcal{T}_{i j, h}$ be uniform mesh square of elements size $h$ on $\omega_{i j}$ which coincides with the boundary $\partial \omega_{i j}^{*}$ and $\partial \omega_{i j}$. The finite element space of bilinear elements on $\mathcal{T}_{i j, h}$ is written $\mathcal{V}_{i j, h}$. Denote by $H_{\mathbb{A}, h}\left(\omega_{i j}^{*}\right)$ the space of discrete $\mathbb{A}$-harmonic functions on $\omega_{i j}^{*}$, i.e., $H_{\mathbb{A}, h}\left(\omega_{i j}^{*}\right)=$ $\left\{\mathbf{v} \in \mathcal{V}_{i j, h}, B(\mathbf{v}, \mathbf{z})=0, \forall \mathbf{z} \in \mathcal{V}_{i j, h}, \mathbf{z}=0\right.$ on $\left.\partial \omega_{i j}^{*}\right\}$. Now the finite element approximation to the spectral problem is to find $\varphi_{i j, h}^{(l)} \in H_{\mathbb{A}, h}\left(\omega_{i j}^{*}\right)$ and $\lambda_{i j, h}^{(l)}$ such that

$$
\left(\varphi_{i j, h}^{(l)}, \chi\right)_{\mathcal{E}\left(\omega_{i} j\right)}=\lambda_{i j, h}^{(l)}\left(\varphi_{i j, h}^{(l)}, \chi\right)_{\mathcal{E}\left(\omega_{i j}^{*}\right)}, \forall \chi \in H_{\mathbb{A}, h}\left(\omega_{i j}^{*}\right) .
$$

Based on the relation between the approximate eigenfunctions $\varphi_{i j, h}^{(l)}$ and the best bilinear finite element approximation of the exact eigenfunctions $\varphi_{i j, h}^{*(l)}$ see, [11], and using the Hypothesis 4.1 and the associated inequalities 4.34 and (4.36) we have

$$
\left\|\varphi_{i j}^{(l)}-\varphi_{i j h}^{(l)}\right\|_{\mathcal{E}\left(\omega_{i j}^{*}\right)} \leq C_{1}(l)\left\|\varphi_{i j}^{(l)}-\varphi_{i j, h}^{*(l)}\right\|_{\mathcal{E}\left(\omega_{i j}^{*}\right)}
$$

and

$$
\left|\lambda_{i j}^{(l)}-\lambda_{i j h}^{(l)}\right| \leq C_{2}(l)\left\|\varphi_{i j}^{(l)}-\varphi_{i j}^{*(l)}\right\|_{\mathcal{E}\left(\omega_{i j}^{*}\right)}^{2} .
$$

Unfortunately the dependence of the constants $C_{1}$ and $C_{2}$ on $l$ is not available. Hence we make the

Hypothesis 5.1. We have that the positive constants $C_{1}$ and $C_{2}$ are bounded above by $C l^{\alpha}$

Applying hypotheses 4.1, 5.1 and (4.36) we get

$$
\left\|\varphi_{i j}^{(l)}-\varphi_{i j h}^{(l)}\right\|_{\mathcal{E}\left(\omega_{i j}^{*}\right)} \leq C H^{-1 / 2} l^{\alpha+\beta} h^{1 / 2}\left\|\varphi_{i j}^{(l)}\right\|_{\mathcal{E}\left(\omega_{i j}^{*}\right)}
$$

and

$$
\left|\lambda_{i j}^{(l)}-\lambda_{i j h}^{(l)}\right|^{1 / 2} \leq C H^{-1 / 2} h^{1 / 2} l^{\alpha+\beta}\left\|\varphi_{i j}^{(l)}\right\|_{\mathcal{E}\left(\omega_{i j}^{*}\right)}
$$


Recalling (5.38) we see that $\mathbf{u}_{0}-\varphi^{p} \in H_{\mathbb{A}}\left(\omega_{i, j}^{*} ; \mathbb{R}^{2}\right)$ can be approximated by local spectral bases so that for $m \sim\left[\ln \left(\tau^{-1}\right)\right]^{3},\left\|\mathbf{u}_{0}-\left(\varphi^{p}+\sum_{l=1}^{m} c_{i j}^{(l)} \varphi_{i j}^{(l)}\right)\right\|_{\mathcal{E}\left(\omega_{i j}\right)} \leq C \tau\left\|\mathbf{u}_{0}-\varphi^{p}\right\|_{\mathcal{E}\left(\omega_{i j}^{*}\right)}$. Applying this we see that

$$
\begin{aligned}
\left\|\left(\mathbf{u}_{0}-\varphi^{p}\right)-\sum_{l=1}^{m} c_{i j}^{(l)} \varphi_{i j, h}^{(l)}\right\|_{\mathcal{E}\left(\omega_{i j}\right)} & \leq\left\|\left(\mathbf{u}_{0}-\varphi^{p}\right)-\sum_{l=1}^{m} c_{i, j}^{(l)} \varphi_{i j}^{(l)}\right\|_{\mathcal{E}\left(\omega_{i j}\right)}+\sum_{l=1}^{m}\left|c_{i j}^{(l)}\right|\left\|\varphi_{i j}^{(l)}-\varphi_{i j, h}^{(l)}\right\|_{\mathcal{E}\left(\omega_{i j}^{*}\right)} \\
& \leq C \tau\left\|\left(\mathbf{u}_{0}-\varphi^{p}\right)\right\|_{\mathcal{E}\left(\omega_{i j}^{*}\right)}+C H^{-1 / 2} h^{1 / 2} \sum_{l=1}^{m}\left|c_{i j}^{(l)}\right| l^{\alpha+\beta} \\
& \leq C \tau\left\|\left(\mathbf{u}_{0}-\varphi^{p}\right)\right\|_{\mathcal{E}\left(\omega_{i j}^{*}\right)}+C H^{-1 / 2} h^{1 / 2} m^{\alpha+\beta}\left(\sum_{l=1}^{\infty} c_{i j}^{(l) 2}\right)^{1 / 2} \\
& \leq C\left(\tau+H^{-1 / 2} h^{1 / 2}\left(\log \tau^{-1}\right)^{3(\alpha+\beta)}\right)\left\|\left(\mathbf{u}_{0}-\varphi^{p}\right)\right\|_{\mathcal{E}\left(\omega_{i j}^{*}\right) .}
\end{aligned}
$$

It now follows that the solution $\mathbf{u}_{0}$ can be approximated on $\omega_{i j}$ by the finite element approximation of the local spectral basis and local particular solution denoted by $\varphi_{h}^{p}$

$$
\left\|\mathbf{u}_{0}-\left(\varphi_{h}^{p}+\sum_{l=1}^{m} c_{i j}^{(l)} \varphi_{i j, h}^{(l)}\right)\right\|_{\mathcal{E}\left(\omega_{i j}\right)} \leq C\left(\tau+H^{-1 / 2} h^{1 / 2}\left(1+\left[\log \tau^{-1}\right]^{3(\alpha+\beta)}\right)\right)\left\|\mathbf{u}_{0}\right\|_{\mathcal{E}\left(\omega_{i j}^{*}\right)}
$$

where $C$ is independent of $\tau, h, H$ and $i, j$. Hence given $\tau$ we can select $h$ to get

$$
\inf _{\left\{c^{(l)}\right\}}\left\|\mathbf{u}_{0}-\left(\varphi_{h}^{p}+\sum c^{(l)} \varphi_{i j, h}^{(l)}\right)\right\|_{\mathcal{E}\left(\omega_{i j}\right)} \leq C \tau\left\|\mathbf{u}_{0}\right\|_{\mathcal{E}\left(\omega_{i j}^{*}\right)}
$$

Let $W_{i, j, h}^{m(i, j)}$ be the span of the functions $\mathbf{v}^{k}, k=1,2,3$ and $\varphi_{i, j, h}^{(l)}, l=0 \ldots, m(i, j)$ augmented with the finite element approximations to the local particular solutions and take $W_{h}^{(m)}=\sum_{i, j} \oplus \phi_{i j} W_{i, j, h}^{(m(i, j))}$. It is now evident that there is an approximation $\mathbf{u}_{0, \text { appr }} \in W_{h}^{(m)}$ for which

$$
\left\|\mathbf{u}_{0}-\mathbf{u}_{0, \operatorname{appr}}\right\|_{\mathcal{E}(\Omega)} \leq C \tau\left\|\mathbf{u}_{0}\right\|_{\mathcal{E}(\Omega)} .
$$

which is the fully discrete analog of (5.39).

We conclude with the following remarks

Remark 5.2. In the estimate (5.46) above we had chosen $h \sim \tau^{2}$ to recover (5.47). This is because we used a uniform mesh and first order finite elements when the eigenfunctions participating in the spectral basis had discontinuous gradient across material interfaces.

Remark 5.3. The eigenfunctions are piecewise analytic inside $\omega^{*}$ and have singular behavior at the points of $\partial \omega^{*}$ where it is intersected by the fiber boundaries. The theory of the eigenvalue problem is very general. It requires only that the distance of the boundaries $\partial \omega^{*}$ and $\partial \omega$ is of the same order as the diameter of $\omega$. Hence we can create a "wiggly" $\omega^{* *}$ when the boundary $\partial \omega^{* *}$ is separated from the fibers by a distance on the order of the fiber diameter and is near by to the boundary of $\omega^{*}$. In this scenario the eigenfunctions belong to the "broken" Sobolev space of arbitrary order. Here a broken Sobolev space is the space which belongs to $H^{1}\left(\omega^{* *}\right)$ and also to $H^{k}$ within the fibers and exterior to the fibers. For this case if we use the curved elements or GFEM/XFEM we can get high order rate of convergence i.e. $N \sim \tau^{-2 /(k-1)}$ where $N$ is the number of degrees of freedom. Note that the exponential decay of the eigenvalues $\lambda^{(l)}$ leads to very mild assumptions about the regularity of the eigenfunctions as put forth in Hypothesis 4.1. 


\section{Computational complexity for MS-GFEM}

In this section the aim is to assess the computational complexity and estimate the cost of the proposed MS-GFEM method for solving the composite material problem. The assessment is qualitative in that it is expressed in terms of constants which are theoretically known but are not necessarily optimal. The computation consists of the following parts: 1) Construction of the uniform mesh of size $\mathrm{H}$ on $\Omega$ over which the subdomains $\omega_{i, j}$ and $\omega_{i . j}^{*}$ are defined. 2) Construction of the spectrally defined shape functions $\varphi_{i j, h}^{l}$ on each subdomain $\omega_{i, j}$. 3) Construction of the stiffness matrix and the right hand side of the MS-GFEM problem. 4) Solving the system of equations of MS-GFEM. Components 1,2,3 can be carried out independently over each subdomain $\omega_{i j}$ and are used to construct the linear system in part 4 . To give perspective on a large parallel machine each subdomain computation can be carried out on a separate core independently of the other cores. The linear system to be solved in part 4 is much smaller in size when compared to the full FE discretization of the problem and can be solved using parallel algorithms.

1. Construction of the coarse mesh of size $H$ and the associated domains $\omega_{i j}, \omega_{i j}^{*}$. This step is straight forward and inexpensive. In what follows we will identify the considerations leading to an optimal choice of $H$ that minimizes the time of computation.

2. The construction of the local shape functions $\varphi_{i j, h}^{l}$ on each $\omega_{i j}$. involves five components.

(a) On $\omega_{i j}^{*}$ we construct the uniform mesh of squares of size $h$ so that the boundaries $\partial \omega_{i j}$ and $\partial \omega_{i j}^{*}$ coincide with the mesh. The size $h$ is chosen according to the tolerance $\tau$ as discussed in the previous section.

(b) Construction of the stiffness matrix for the problem on $\omega_{i j}^{*}$. From the previous section these domains are squares of sidelength $\beta(1+\alpha) H=H^{*}$. Because the tensor $\mathbb{A}$ is discontinuous across material interfaces we point out that adaptive integration schemes need to be used. Because of the sparsity of the stiffness matrix the number of operations required for assembly is $\mathcal{O}\left(H^{*} / h\right)$.

(c) Construction of the space $H_{\mathbb{A}, h}\left(\omega_{i j}^{*}\right)$ of discrete $\mathbb{A}$-harmonic functions on $\omega_{i j}^{*}$. Motivated by the numerical experiments of [55] we construct a $2 N$ dimensional space of discrete finite element $\mathbb{A}$-harmonic functions spanned by the functions $\left\{w_{i j, h}^{l}\right\}$ on $\omega_{i j}^{*}$. These functions are defined to be discretely $\mathbb{A}$-harmonic with traces on $\partial \omega_{i j}^{*}$ corresponding to the basis of harmonic polynomials of degree $\leq N$. The choice of $N$ is determined at the time of computation and is conditioned on the eigenvalue $\lambda_{i j, h}^{(2 N+1)}$ of the discrete spectral problem (5.40). Here we choose $N$ such that $\lambda_{i j, h}^{(2 N+1)} \leq \tau$. We then select the dimension of the discrete approximation space to be $\mathrm{m}$ such that $\lambda_{i j, h}^{2 m+1} \leq \tau$. For the purposes of estimation we start with $N=100$. The functions $w_{i j, h}^{l}$ can be computed iteratively using conjugate gradient methods and preconditioning or by Gaussian elimination. Here we will assume that each of the discrete functions $w_{i j, h}^{l}$ is obtained iteratively using $q$ iterations so that $\mathcal{O}\left(2 N q(H / h)^{2}\right)$ operations are required to construct a discrete $2 N$ dimensional harmonic subspace of $H_{\mathbb{A}, h}\left(\omega_{i j}^{*}\right)$. Alternatively using Gaussian elimination would require $\mathcal{O}\left((2 N H / h)^{3}\right)$ operations to create the $2 N$ dimensional subspace.

(d) Construction of the eigenvalue problem leading to the local shape functions $\varphi_{i j, h}^{l}$. The local discrete eigenvalue problem (5.40) delivers the matrix eigenvalue problem of the form $\lambda P \mathbf{x}=Q \mathbf{x}$. Here $P_{k l}=$ $\left(w_{i j, h}^{k}, w_{i j, h}^{l}\right)_{\omega_{i j}^{*}}$, and noting that the local basis functions $w_{i h, h}^{k}$ and $w_{i j, h}^{l}$ are $\mathbb{A}$-harmonic shows (on integrating by parts) that computing one element of $P$ requires $\mathcal{O}(H / h)$ operations. Hence $\mathcal{O}\left(4 N^{2} H / h\right)$ operations are required to assemble $P$. The same estimate applies to the number of operations needed to assemble $Q$.

(e) Solution of the eigenvalue problem requires $\mathcal{O}\left((2 N)^{3}\right)$ operations.

Summarizing we see that to obtain the approximation space spanned by $2 N$ local shape functions given by the spectral basis $\left\{\varphi_{i j}^{l}\right\}_{l=1}^{2 N}$ the cost is dominated by the number of operations required to construct the finite dimensional discrete subspace spanned by $\left\{w_{i j, h}^{l}\right\}$. This cost is $\mathcal{O}\left(2 N q(H / h)^{2}\right)$ operations using the iterative approach for finding each $w_{i j, h}^{l}$ and $\mathcal{O}\left((2 N H / h)^{3}\right)$ operations using Gaussian elimination. Assuming that $q=N$ we need $\mathcal{O}\left(2 N^{2}(H / h)^{2}\right)$ respectively $\mathcal{O}\left(8 N^{3} H^{3} / h^{3}\right)$ operations for determining $\left\{\varphi_{i j}^{l}\right\}_{l=1}^{2 N}$. 
3. Construction of the MS-GFEM stiffness matrix and the right hand side,

(a) Construction of the MS-GFEM stiffness matrix. The MS-GFEM stiffness matrix is a block diagonal matrix associated with the 9 point stencil. Here each block is an $m \times m$ matrix. The construction of the block matrices is carried out locally and can be done in parallel. Here each stencil requires $\mathcal{O}\left(m^{2}(H / h)^{2}\right)$ operations to assemble and we have $(\kappa / H)^{2}$ stencils.

(b) Construction of the right hand side. For problems driven by body forces one has a prescribed right hand side function $f$. For this case we compute $(\kappa / H)^{2}$ particular solutions $w_{i j}$. Here we need $\left.\mathcal{O}(H / h)^{2}\right)$ operations to compute each local particular solution $\mathbf{w}_{i j}$. To compute all local particular solutions associated with a right hand side we need $\left.\mathcal{O}(\kappa H / h)^{2}\right)$ operations.

4. Solution of the MS-GFEM system. For problems with multiple right hand sides we can invert the matrix using elimination to recover the discrete solution operator. This requires $\mathcal{O}\left((\kappa / H)^{3} m^{3}\right)$ operations. The solution procedure is parallelizable but requires communication between cores. Alternatively for single right hand sides one can proceed using iterative methods.

We now address the computational complexity for the MS-GFEM and estimate the machine time required for computation. To begin we consider implementation first on a single processor and then on a parallel machine with $p$ processors. Here we ignore other issues such as the cost of fetches to disk when using a single processor.

1. Single processor implementation. Carrying out steps 1 through 3 requires $\mathcal{O}\left(N^{2} H^{2} / h^{2}\right)$ operations for each of the $(\kappa / H)^{2}$ subdomains $\omega_{i j}$ for a total of $\mathcal{O}\left(\kappa^{2} N^{2} / h^{2}\right)$ operations. If we apply elimination to construct the local subspaces then $\mathcal{O}\left(N^{3} H^{3} / h^{3}\right)$ operations are required over each subdomain and we have a total of $\mathcal{O}\left(\kappa^{2} N^{3} H / h^{3}\right)$ operations. Thus to to carry out steps 1 through 3 on a single processor requires $\mathcal{O}\left(\kappa^{2} N^{2} / h^{2}\right)$ resp $\mathcal{O}\left(\kappa^{2} N^{3} H / h^{3}\right)$ operations. Step 4 requires $\mathcal{O}\left(m^{3} \kappa^{3} / H^{3}\right)$ operations. It follows that single processor implementation of MS-GFEM requires

$$
\begin{array}{r}
\mathcal{O}\left(\kappa^{2} N^{2} / h^{2}\right)+\mathcal{O}\left(\kappa^{3} m^{3} / H^{3}\right) \text { and } \\
\mathcal{O}\left(\kappa^{2} N^{3} H / h^{3}\right)+\mathcal{O}\left(m^{3} \kappa^{3} / H^{3}\right)
\end{array}
$$

operations respectively. Fixing $h, N, m$ in (6.49) and choosing $H$ so that both terms are of the same order we have $H \sim \mathcal{O}\left(\kappa^{1 / 3} m h^{2 / 3} / N^{2 / 3}\right)$ and the total number of operations is $\mathcal{O}\left(\kappa^{2} N^{2} / h^{2}\right)$. Similarly fixing $h$ in (6.50) and choosing $H$ so that both terms are of the same order gives resp $H=m^{3 / 4} \kappa^{1 / 4} h^{3 / 4} / N^{3 / 4}$ and the total number of operations is $\mathcal{O}\left(N^{9 / 4} m^{1 / 4} \kappa^{9 / 4} / h^{9 / 4}\right)$.

Equation (6.49) shows that the local approximation spaces together with the inversion of the GFEM stiffness matrix delivers the complete inverse operator for the discrete problem with the same number $\mathcal{O}\left(N^{2} \kappa^{2} / h^{2}\right)$ of operations.

2. Now we consider a parallel implementation with $p$ processors and provide an estimate for the time necessary to perform the computation. Here the work is measured in floating point operations per second $\mathcal{F}$ and the total time is $\mathcal{F} \times$ Operations. Here the time to execute steps one through three drops by a factor of $\frac{1}{p}$. Step four using a direct solver within a parallel implementation will roughly require $\mathcal{F} \mathcal{O}\left(\kappa^{3} m^{3} / H^{3}\right) / p+$ $D(\lg p) m(\kappa / H)+E(m \kappa / H)^{2} p^{-1 / 2}$ s. Here $D \sim 10^{-6}$ and $E \sim 10^{-9}$ and the last two terms relate to the latency inherent in the direct solver.

We emphasize that the implementation of MS-GFEM delivers the discrete solution operator using the same order of operations as the number of fibers inside the computational domain. This implementation is optimal in that the number of operations for solution is of the same order as the input data for the problem. It is important to point out that the MS-GFEM matrix is of order $m^{2} \kappa^{2} / H^{2}$, where $m=\ln ^{d+1}\left(\frac{1}{\tau}\right)$ where $\tau$ is the prescribed tolerance for the approximation error hence the memory required to store the MS-GFEM inverse matrix can be controlled and can be of order far less than the number of fibers contained within the computational domain.

We close this section with a discussion on local memory allocation and problem size. Here we execute steps 1 through 3 of the MS-GFEM computation over a single patch $\omega_{i j}^{*}$ on one processor. Standard processors and 
associated cash memory can handle problems with $(H / h)^{2} \leq 10^{6}$ degrees of freedom. For this scenario the time for the local computation will be on the order of one second. Now suppose that $h=\tau^{2}$ then we can choose $H=10^{3} \tau^{2}$ and for $\tau=10^{-2}$ we have $H \sim 10^{-1}$. Setting $\kappa=1$ we have $p=10^{2}$ processors and the size of the MS-GFEM inverse matrix is seen to be on the order of $10^{5}$. Assuming that the number of fibers is proportional to $\frac{1}{h^{2}}$ we have $10^{8}$ fibers in the computational domain. Thus the MS-GFEM inverse matrix is three orders of magnitude smaller than the number of fibers inside the computational domain.

\section{Optimal local approximation SpaCes}

In this section we introduce and develop optimal local approximation spaces for use in the multi-scale scheme. These spaces are distinguished by their exponential approximation properties. Given the solution $\mathbf{u}_{0}$ to the global problem and a prescribed tolerance $\tau$ we seek to find a local approximation on the patch $\omega_{j}$. In this section we show that is is possible to find a local approximation $\mathbf{w}$ from the optimal local space $\Psi_{j}$ of dimension $\left(\ln \tau^{-1}\right)^{d+1}$, $d=2,3$ for which the error satisfies

$$
\left\|\mathbf{u}_{0}-\mathbf{w}\right\|_{\mathcal{E}\left(\omega_{j}\right)} \leq \tau
$$

In what follows we will establish the optimal local approximation properties in the general context of heterogeneous media for two and three dimensional elasticity problems characterized by measurable elasticity tensors $\mathbb{A}(\mathbf{x})$ satisfying the coercivity and boundedness conditions (2.3). We will also develop approximation properties for local domains that border the boundary of $\Omega$. Here we will establish exponential approximation results when $\Omega$ has reentrant corners and for general Lipschitz domains. We begin by identifying optimal local approximation spaces for interior domains. To fix ideas we will assume that the patch $\omega$ is a cube of a given side length surrounded by a larger cube $\omega^{*}$. We will distinguish two cases depending on if the set $\omega$, lies within the interior of $\Omega$ or if it intersects the boundary, i.e., $\bar{\omega} \cap \partial \Omega \neq \emptyset$. It will be shown that the overall approach to constructing optimal local approximation spaces for these two cases is the same. We consider concentric cubes $\omega \subset \omega^{*}$ with side lengths given by $\sigma$ and $\sigma^{*}=(1+\rho) \sigma$ respectively. In order to introduce the ideas we suppose first that $\omega$ lies in the interior of $\Omega$ so that $\omega \subset \omega^{*} \subset \Omega$.

We shall utilize $\omega^{*}$ to construct a finite dimensional approximation space over $\omega$. For any open subset $S$ of the computational domain $\Omega$ we introduce the space of functions $H_{\mathbb{A}}\left(S ; \mathbb{R}^{d}\right)$ defined to be the functions in $H^{1}\left(S ; \mathbb{R}^{d}\right)$ that are $\mathbb{A}$-harmonic on $S$, i.e., $\mathbf{v} \in H^{1}\left(S ; \mathbb{R}^{d}\right)$ and

$$
(\mathbf{v}, \varphi)_{\mathcal{E}(S)}=\int_{S} \mathbb{A} e(\mathbf{v}): e(\varphi) \mathrm{d} x, \quad \forall \varphi \in C_{0}^{\infty}\left(S ; \mathbb{R}^{d}\right)
$$

Here $H_{\mathbb{A}}\left(\omega ; \mathbb{R}^{d}\right)$ and $H_{\mathbb{A}}\left(\omega^{*} ; \mathbb{R}^{d}\right)$ contain local information on the heterogeneities and will be used in the construction of the optimal local basis. Let $\mathcal{R}=\left\{\mathbf{a}+\mathbf{b} \wedge \mathbf{x} ; \mathbf{a}\right.$ and $\mathbf{b}$, in $\left.\mathbb{R}^{d}\right\}$ be the linear space of rigid motions. We introduce the quotient of $H_{\mathbb{A}}\left(\omega^{*} ; \mathbb{R}^{d}\right) / \mathcal{R}$ with respect to $\mathcal{R}$ denoted by $H_{\mathbb{A}}\left(\omega^{*} ; \mathbb{R}^{d}\right) / \mathcal{R}$. It is clear that the solution $\mathbf{u}$ lies in this space. We also introduce the subspace $H_{\AA}^{0}\left(\omega^{*} ; \mathbb{R}^{d}\right)$ given by elements of $H_{\mathbb{A}}\left(\omega^{*} ; \mathbb{R}^{d}\right)$ perpendicular to $\mathcal{R}$ with respect to the $L^{2}\left(\omega^{*} ; \mathbb{R}^{d}\right)$ inner product. Here we recall that $H_{\mathbb{A}}\left(\omega^{*} ; \mathbb{R}^{d}\right) / \mathcal{R}$ equipped with the energy inner product is isometric to $H_{\AA}^{0}\left(\omega^{*} ; \mathbb{R}^{d}\right)$. The closure of smooth functions with compact support in the energy norm is denoted by $H_{0}^{1}\left(\omega^{*} ; \mathbb{R}^{d}\right)$ and for future reference we introduce the decomposition of $H^{1}\left(\omega^{*} ; \mathbb{R}^{d}\right)$ given by

$$
H^{1}\left(\omega^{*} ; \mathbb{R}^{d}\right)=H_{\mathbb{A}}^{0}\left(\omega^{*} ; \mathbb{R}^{d}\right) \oplus H_{0}^{1}\left(\omega^{*} ; \mathbb{R}^{d}\right) \oplus \mathcal{R} .
$$

Here $H_{\AA}^{0}\left(\omega^{*} ; \mathbb{R}^{d}\right)$ and $H_{0}^{1}\left(\omega^{*} ; \mathbb{R}^{d}\right)$ are orthogonal with respect to the energy inner product on $\omega^{*}$.

We choose to approximate elements in the space of functions $H_{\mathbb{A}}\left(\omega^{*} ; \mathbb{R}^{d}\right) / \mathcal{R}$ restricted to $\omega$. Let $\mathcal{T}$ : $H_{\mathbb{A}}\left(\omega^{*} ; \mathbb{R}^{d}\right) / \mathcal{R} \rightarrow H_{\mathbb{A}}\left(\omega ; \mathbb{R}^{d}\right) / \mathcal{R}$ be the restriction operator such that $\mathcal{T}(\mathbf{u})(x)=u(x)$ for all $\mathbf{x} \in \omega$ and $\mathbf{u} \in H_{\mathbb{A}}\left(\omega^{*} ; \mathbb{R}^{d}\right) / \mathcal{R}$. The operator $\mathcal{T}$ is compact, this follows immediately from an application of the the Caccioppoli inequality Lemma 7.6 together with the Rellich Kondrachov embedding theorem on $\omega^{*}$ see [7]. 
Now we approximate by " $n$ " dimensional subspaces $S(n) \subset H_{\mathbb{A}}\left(\omega ; \mathbb{R}^{d}\right) / \mathcal{R}$. The accuracy of a particular increasing sequence $\{S(n)\}_{n=1}^{\infty}$ of local approximation spaces is measured by

$$
d(S(n), \omega)=\sup _{\mathbf{u} \in H_{\mathbb{A}}\left(\omega^{*} ; \mathbb{R}^{d}\right) / \mathcal{R}} \inf _{\chi \in S(n)} \frac{\|\mathcal{T} \mathbf{u}-\chi\|_{\mathcal{E}(\omega)}}{\|\mathbf{u}\|_{\mathcal{E}\left(\omega^{*}\right)}}
$$

A sequence of approximation spaces $\hat{S}(n)$ is said to be optimal if it has an accuracy $d(\hat{S}(n), \omega)$ that satisfies $d(\hat{S}(n), \omega) \leq d(S(n), \omega), n=1,2, \ldots$, when compared to any other sequence of approximation spaces $S(n)$. The problem of finding the family of optimal local approximation spaces is formulated as follows. Let

$$
d_{n}\left(\omega, \omega^{*}\right)=\inf _{S(n)} \sup _{\mathbf{u} \in H_{\mathbb{A}}\left(\omega^{*} ; \mathbb{R}^{d}\right) / \mathcal{R}} \inf _{\chi \in S(n)} \frac{\|\mathcal{T} \mathbf{u}-\chi\|_{\mathcal{E}(\omega)}}{\|\mathbf{u}\|_{\mathcal{E}\left(\omega^{*}\right)}}
$$

Then the optimal family of approximation spaces $\left\{\Psi_{n}(\omega)\right\}_{n=1}^{\infty}$ satisfy

$$
d_{n}\left(\omega, \omega^{*}\right)=\sup _{\mathbf{u} \in H_{\mathbb{A}}\left(\omega^{*} ; \mathbb{R}^{d}\right) / \mathcal{R}} \inf _{\chi \in \Psi_{n}(\omega)} \frac{\|\mathcal{T} \mathbf{u}-\chi\|_{\mathcal{E}(\omega)}}{\|\mathbf{u}\|_{\mathcal{E}\left(\omega^{*}\right)}}
$$

The quantity $d_{n}\left(\omega, \omega^{*}\right)$ is known as the Kolomogorov n-width of the compact operator $\mathcal{T}$, see [45]. The optimal local approximation space $\Psi_{n}(\omega)$ for GFEM follows from general considerations. We introduce the adjoint operator $\mathcal{T}^{*}: H_{\mathbb{A}}\left(\omega ; \mathbb{R}^{d}\right) / \mathcal{R} \rightarrow H_{\mathbb{A}}\left(\omega^{*} ; \mathbb{R}^{d}\right) / \mathcal{R}$ and the operator $\mathcal{T}^{*} \mathcal{T}$ is a compact, self adjoint, non-negative operator mapping $H_{\mathbb{A}}\left(\omega^{*} ; \mathbb{R}^{d}\right) / \mathcal{R}$ into itself. We denote the eigenfunctions and eigenvalues of the problem

$$
\mathcal{T}^{*} \mathcal{T} \mathbf{u}=\lambda \mathbf{u}
$$

by $\left\{\varphi_{i}\right\}$ and $\left\{\lambda_{i}\right\}$ and the optimal subspace $\Psi_{n}$ is given by the following theorem.

Theorem 7.1. The optimal approximation space is given by $\Psi_{n}(\omega)=\operatorname{span}\left\{\psi_{1}, \ldots, \psi_{n}\right\}$, where $\psi_{i}=\mathcal{T} \varphi_{i}$ and $d_{n}\left(\omega, \omega^{*}\right)=\sqrt{\lambda_{n+1}}$.

For the case considered here the definitions of $\mathcal{T}$ and $\mathcal{T}^{*}$ show that the optimal subspace and eigenvalues are given by the following explicit eigenvalue problem.

Theorem 7.2. The optimal approximation space is given by $\Psi_{n}(\omega)=\operatorname{span}\left\{\psi_{1}, \ldots, \psi_{n}\right\}$ where $\psi_{i}=\mathcal{T} \varphi_{i}$ and $\varphi_{i}$ and $\lambda_{i}$ are the first $n$ eigenfunctions and eigenvalues that satisfy

$$
\left(\varphi_{i}, \delta\right)_{\mathcal{E}(\omega)}=\lambda_{i}\left(\varphi_{i}, \delta\right)_{\mathcal{E}\left(\omega^{*}\right)}, \forall \delta \in H_{\mathbb{A}}\left(\omega^{*} ; \mathbb{R}^{d}\right) / \mathcal{R} .
$$

The next theorem provides an upper bound on the rate of convergence for the optimal local approximation.

Theorem 7.3. Exponential convergence for interior approximations.

For $\epsilon>0$ there is an $N_{\epsilon}>0$ such that for all $n>N_{\epsilon}$

$$
d_{n}\left(\omega, \omega^{*}\right) \leq \mathrm{e}^{-n\left(\frac{1}{1+d}-\epsilon\right)} .
$$

Theorem 7.3 (proven below) shows that the asymptotic convergence rate associated with the optimal approximation space is nearly exponential for the general class of $L^{\infty}(\Omega)$ coefficients satisfying the coercivity and boundedness conditions (2.3).

We now present optimal local approximation spaces for domains bordering the boundary of $\Omega$. These results hold for domains $\Omega$ of general shape including bounded Lipschitz regions. The essential assumption is that the 
Rellich Kondrachov embedding theorem holds in $\Omega$. To fix ideas we consider the $L$ shaped domain $\Omega$ with a reentrant corner and introduce optimal local approximation spaces for domains that intersect the boundary $\partial \Omega$. To illustrate the method consider concentric $L$ shaped subdomains $\omega \subset \omega^{*}$ of $\Omega$ containing the reentrant corner. See Figure 3. The arguments presented here naturally apply to other choices of $\omega$ and $\omega^{*}$ that touch the boundary. The outer domain is denoted by $\omega^{*}$ and the concentric inner domain is denoted by $\omega$. The side lengths of $\omega \subset \omega^{*} \subset \sigma$ are given by $\sigma$ and $\sigma^{*}=(1+\rho) \sigma$ respectively.

Given a function $\mathbf{u} \in H_{\mathbb{A}}\left(\Omega ; \mathbb{R}^{d}\right), d=2,3$, the goal is to provide a approximation to $\mathbf{u}$ in $\omega$. To this end we form a local particular solution $\mathbf{u}_{p}$ given by the $\mathbb{A}$-harmonic function that satisfies $\mathbb{A} e\left(\mathbf{u}_{p}\right) \mathbf{n}=\mathbf{g}$ on $\partial \omega^{*} \cap \partial \Omega$ and $\mathbf{u}_{p}=0$ on $\partial \omega^{*} \cap \Omega$. Writing $\mathbf{u}=\mathbf{u}_{p}+\mathbf{u}_{0}$ we see that $\mathbb{A} e\left(u_{0}\right) \mathbf{n}=0$ on $\partial \omega^{*} \cap \partial \Omega$ and $\mathbf{u}_{0}=\mathbf{u}$ on $\partial \omega^{*} \cap \Omega$. The objective of this section is to find the optimal family of local approximation spaces that give the best approximation to $\mathbf{u}_{0}=\mathbf{u}-\mathbf{u}_{p}$ in the energy norm over the set $\omega$. We introduce the space of functions $H_{\mathbb{A}, 0}\left(\omega^{*} ; \mathbb{R}^{d}\right)$ given by all functions $\mathbf{v} \in H^{1}\left(\omega^{*}, \mathbb{R}^{d}\right)$ that are $\mathbb{A}$-harmonic on $\omega^{*}$ and for which $\partial_{\nu} \mathbf{v} \equiv \mathbb{A} e(\mathbf{v}) \mathbf{n}=0$ on $\partial \omega^{*} \cap \partial \Omega$. The analogous space of functions defined on $\omega$ is denoted $H_{\mathbb{A}, 0}\left(\omega ; \mathbb{R}^{d}\right)$. Since we approximate functions with respect to the energy norm we introduce the quotient space of $H_{\mathbb{A}, 0}\left(\omega^{*} ; \mathbb{R}^{d}\right)$ to the subspace of rigid translations $\mathcal{R}$ denoted by $H_{\mathbb{A}, 0}\left(\omega^{*} ; \mathbb{R}^{d}\right) / \mathcal{R}$. Now we introduce $\mathcal{T}: H_{\mathbb{A}, 0}\left(\omega^{*} ; \mathbb{R}^{d}\right) / \mathcal{R} \rightarrow H_{\mathbb{A}, 0}\left(\omega ; \mathbb{R}^{d}\right) / \mathcal{R}$ given by the restriction operator defined by $\mathcal{T}(\mathbf{u})(\mathbf{x})=\mathbf{u}(\mathbf{x})$ for all $\mathbf{x} \in \omega$ and $\mathbf{u} \in H_{\mathbb{A}, 0}\left(\omega^{*} ; \mathbb{R}^{d}\right) / \mathcal{R}$. The operator $\mathcal{T}$ is compact. As before this follows immediately from an application of a suitable Caccioppoli inequality (Lem. 7.6) together with the Rellich Kondrachov embedding theorem on $\omega^{*}$. Let $S(n)$ be any finite dimensional subspace of $H_{\mathbb{A}, 0}\left(\omega ; \mathbb{R}^{d}\right) / \mathcal{R}$ and the problem of finding the family of optimal local approximation spaces is formulated in terms of the n-width of $\mathcal{T}$. Let

$$
d_{n}\left(\omega, \omega^{*}\right)=\inf _{S(n)} \sup _{\mathbf{u} \in H_{\mathrm{A}, 0}\left(\omega^{*} ; \mathbb{R}^{d}\right) / \mathcal{R}} \inf _{\chi \in S(n)} \frac{\|\mathcal{T} \mathbf{u}-\chi\|_{\mathcal{E}(\omega)}}{\|\mathbf{u}\|_{\mathcal{E}\left(\omega^{*} \cap \Omega\right)}}
$$

Proceeding as before we introduce the adjoint operator $\mathcal{T}^{*}: H_{\mathbb{A}}\left(\omega ; \mathbb{R}^{d}\right) / \mathcal{R} \rightarrow H_{\mathbb{A}}\left(\omega^{*}\right) / \mathcal{R}$ and the operator $\mathcal{T}^{*} \mathcal{T}$ is a compact operator mapping $H_{\mathbb{A}, 0}\left(\omega^{*}\right) / \mathcal{R}$ into itself. The optimal approximating spaces are described in the following theorem.

Theorem 7.4. The optimal approximation space is given by $\Psi_{n}(\omega)=\operatorname{span}\left\{\psi_{1}, \ldots, \psi_{n}\right\}$ where $\psi_{i}=\mathcal{T} \varphi_{i}$ and $\varphi_{i} \in H_{\mathbb{A}, 0}\left(\omega^{*} ; \mathbb{R}^{d}\right) / \mathcal{R}$ and $\lambda_{i}$ are the first $n$ eigenfunctions and eigenvalues that satisfy

$$
\left(\varphi_{i}, \delta\right)_{\mathcal{E}(\omega)}=\lambda_{i}\left(\varphi_{i}, \delta\right)_{\mathcal{E}\left(\omega^{*} \cap \Omega\right)}, \forall \delta \in H_{\mathbb{A}, 0}\left(\omega^{*} ; \mathbb{R}^{d}\right) / \mathcal{R},
$$

and $d_{n}\left(\omega, \omega^{*}\right)=\sqrt{\lambda_{n+1}}$.

The next theorem provides an upper bound on the rate of convergence for the optimal local boundary approximation.

Theorem 7.5 (Exponential convergence at the boundary).

For $\epsilon>0$ there is an $N_{\epsilon}>0$ such that for all $n>N_{\epsilon}$

$$
d_{n}\left(\omega, \omega^{*}\right) \leq \mathrm{e}^{-n\left(\frac{1}{d+1}-\epsilon\right)} .
$$

Theorem 7.5 shows that the asymptotic convergence rate associated with the optimal boundary approximation space is nearly exponential for the general class of $L^{\infty}\left(\omega^{*}\right)$ coefficients $\mathbb{A}(\mathbf{x})$ satisfying the coercivity and boundedness conditions (2.3).

We provide a proof of exponential decay for interior subdomains noting that the proof for boundary domains proceeds along similar lines. The construction of the local approximation space is done iteratively. We introduce the the first $n$ eigenfunctions $\mathbf{v}_{i} \in H^{1}\left(\omega^{*} ; \mathbb{R}^{d}\right)$, orthogonal to $\mathcal{R}$, in the $L^{2}\left(\omega^{*} ; \mathbb{R}^{d}\right)$ inner product, for the the eigenvalue problem

$$
\left(\mathbf{v}_{i}, \mathbf{w}\right)_{\mathcal{E}\left(\omega^{*}\right)}=\lambda_{i} \int_{\omega^{*}} \mathbf{v}_{i} \cdot \mathbf{w} \mathrm{d} x, \forall \mathbf{w} \in H^{1}\left(\omega^{*} ; \mathbb{R}^{d}\right)
$$


$i=1, \ldots, n$. The subspace spanned by these functions is denoted by $S_{n}\left(\omega^{*}\right)$. We introduce the span of $\mathbb{A}$ harmonic functions given by

$$
W_{n}\left(\omega^{*}\right)=\operatorname{span}\left\{\mathbf{w}_{i} \in H_{\mathbb{A}}\left(\omega^{*} ; \mathbb{R}^{d}\right): w_{i}=\mathbf{v}_{i}, \text { on } \partial \omega^{*}, i=1, \ldots n\right\} .
$$

The orthogonal projection from $H^{1}\left(\omega^{*} ; \mathbb{R}^{d}\right)$ onto $H_{A}^{0}\left(\omega^{*} ; \mathbb{R}^{d}\right)$ is denoted by $\mathcal{P}^{\mathbb{A}}$. and one readily checks that $W_{n}\left(\omega^{*}\right)=\mathcal{P}^{\mathbb{A}} S_{n}\left(\omega^{*}\right)$.

We define the family of approximation spaces $\mathcal{F}_{n}\left(\omega, \omega^{*}\right)$ given by the restriction of the elements of $W_{n}\left(\omega^{*}\right)$ to $\omega$. In what follows we first show that $\mathcal{F}_{n}\left(\omega, \omega^{*}\right)$ is a family of local approximation spaces with a rate of convergence on the order of $n^{-1 / d}, d=2,3$. To show this we introduce a suitable version of the Caccioppoli inequality that bounds functions in the energy norm over any measurable subset $\mathcal{O} \subset \omega^{*}$ for which $\operatorname{dist}\left(\partial \mathcal{O}, \partial \omega^{*}\right)>\delta>0$ in terms of the $L^{2}$ norm over $\omega^{*}$.

Lemma 7.6 (Caccioppoli inequality).

Let $\mathbf{u}$ be $\mathbb{A}$-harmonic in $\omega^{*}$ and belong to $L^{2}\left(\omega^{*} ; \mathbb{R}^{d}\right) \cap H_{l o c}^{1}\left(\omega^{*} ; \mathbb{R}^{d}\right)$. Then

$$
\|\mathbf{u}\|_{\mathcal{E}(\mathcal{O})} \leq\left(2(\beta)^{1 / 2} / \delta\right)\|\mathbf{u}\|_{L^{2}\left(\omega^{*} ; \mathbb{R}^{d}\right)}
$$

where $\beta$ is defined in (2.3).

The proof of the Lemma follows that given in [7].

Next we introduce the approximation theorem associated with the space $W_{n}\left(\omega^{*}\right)$ given by

Lemma 7.7. Let $\mathbf{u} \in H_{A}^{0}\left(\omega^{*} ; \mathbb{R}^{d}\right)$ then there exists a $\mathbf{v}_{\mathbf{u}} \in W_{n}\left(\omega^{*}\right)$ such that

$$
\left\|\mathbf{u}-\mathbf{v}_{\mathbf{u}}\right\|_{L^{2}\left(\omega^{*} ; \mathbb{R}^{d}\right)}=\inf _{\mathbf{v} \in W_{n}\left(\omega^{*}\right)}\|\mathbf{u}-\mathbf{v}\|_{L^{2}\left(\omega^{*} ; \mathbb{R}^{d}\right)} \leq C_{n} \sigma^{*} \theta_{n} \alpha^{-1 / 2}\|\mathbf{u}\|_{\mathcal{E}\left(\omega^{*}\right)} \quad d=2,3,
$$

where $\sigma^{*}$ is the side length of the cube $\omega^{*}, \alpha$ is given by (2.3) $C_{n}=n^{-1 / d}(1+o(1)), d=2,3$. For $d=2$, $\theta_{2}=\sqrt{2 /(3 \pi)}$ and for $d=3, \theta_{3}=\left[(1+4 \sqrt{2}) /\left(6 \pi^{2}\right)\right]^{1 / 3}$.

Proof. The lemma follows immediately from an upper bound on the quotient

$$
T=\sup _{\mathbf{u} \in \in H_{\AA}^{0}\left(\omega^{*} ; \mathbb{R}^{d}\right)} \inf _{\mathbf{w} \in W_{n}\left(\omega^{*}\right)} \frac{\|\mathbf{u}-\mathbf{w}\|_{L^{2}\left(\omega^{*} ; \mathbb{R}^{d}\right)}}{\|\mathbf{u}\|_{\mathcal{E}\left(\omega^{*}\right)}}
$$

Fix $\mathbf{u} \in H_{\AA}^{0}\left(\omega^{*} ; \mathbb{R}^{d}\right)$ and denote the projection of $\mathbf{u}$ onto $W_{n}\left(\omega^{*}\right)$ with respect to the energy norm $\|\cdot\|_{\mathcal{E}\left(\omega^{*}\right)}$ by $\mathcal{P}^{\mathcal{E}} \mathbf{u}$. Choosing $\mathbf{w}=\mathcal{P}^{\mathcal{E}} \mathbf{u}$ and noting that $\left\|\left(I-\mathcal{P}^{\mathcal{E}}\right) \mathbf{u}\right\|_{\mathcal{E}\left(\omega^{*}\right)} \leq\|\mathbf{u}\|_{\mathcal{E}\left(\omega^{*}\right)}$ gives the upper bound

$$
T \leq \sup _{\mathbf{u} \in H_{A}^{0}\left(\omega^{*} ; \mathbb{R}^{d}\right)} \frac{\left\|\left(I-\mathcal{P}^{\mathcal{E}}\right) \mathbf{u}\right\|_{L^{2}\left(\omega^{*} ; \mathbb{R}^{2}\right)}}{\|(I-\mathcal{P} \mathcal{E}) \mathbf{u}\|_{\mathcal{E}\left(\omega^{*}\right)}}=\sup _{\mathbf{u} \in H_{\AA}^{0}\left(\omega^{*} ; \mathbb{R}^{d}\right) \perp W_{n}\left(\omega^{*}\right)} \frac{\|\mathbf{u}\|_{L^{2}\left(\omega^{*} ; \mathbb{R}^{2}\right)}}{\|\mathbf{u}\|_{\mathcal{E}\left(\omega^{*}\right)}}
$$

Since $W_{n}\left(\omega^{*}\right)=\mathcal{P}^{\mathbb{A}} S_{n}\left(\omega^{*}\right)$ it follows that

$$
\begin{array}{r}
\left\{\mathbf{u} \in H_{\mathbb{A}}^{0}\left(\omega^{*} ; \mathbb{R}^{d}\right) \perp \mathcal{P}^{\mathbb{A}} S_{n}\left(\omega^{*}\right)\right\}=\left\{\mathbf{u} \in H_{A}^{0}\left(\omega^{*} ; \mathbb{R}^{d}\right) \perp S_{n}\left(\omega^{*}\right)\right\}, \\
\left\{\mathbf{u} \in H_{\mathbb{A}}^{0}\left(\omega^{*} ; \mathbb{R}^{d}\right) \perp S_{n}\left(\omega^{*}\right)\right\} \subset\left\{u \in H^{1}\left(\omega^{*} ; \mathbb{R}^{d}\right)^{2} \perp_{L^{2}}\left(S_{n}\left(\omega^{*}\right) \oplus \mathcal{R}\right)\right\},
\end{array}
$$

where $\perp_{L^{2}}$ in the second line of (7.67) denotes orthogonality with respect to the $L^{2}\left(\omega^{*} ; \mathbb{R}^{d}\right)$ inner product. Hence

$$
T \leq \sup _{\mathbf{u} \in H_{\AA}^{0}\left(\omega^{*} ; \mathbb{R}^{d}\right) \perp S_{n}\left(\omega^{*}\right)} \frac{\|\mathbf{u}\|_{L^{2}\left(\omega^{*} ; \mathbb{R}^{d}\right)}}{\|\mathbf{u}\|_{\mathcal{E}\left(\omega^{*}\right)}} \leq \sup _{\mathbf{u} \in H^{1}\left(\omega^{*} ; \mathbb{R}^{d}\right)^{2} \perp_{L^{2}}\left(S_{n}\left(\omega^{*}\right) \oplus R\right)} \frac{\|\mathbf{u}\|_{L^{2}\left(\omega^{*}\right)}}{\|\mathbf{u}\|_{\mathcal{E}\left(\omega^{*}\right)}}=\frac{1}{\sqrt{\mu_{n+1}}}
$$


where $\mu_{n+1}$ is the largest eigenvalue associated with $S_{n+1}\left(\omega^{*}\right)$. One has the elementary lower bound $\mu_{n+1} \geq$ $\alpha \nu_{n+1}$ where $\nu_{n+1}$ is the largest corresponding eigenvalue for $\int_{\omega^{*}} e\left(\mathbf{v}_{n+1}\right): e(\mathbf{w}) \mathrm{d} x=\nu_{n+1} \int_{\omega^{*}} \mathbf{v}_{n+1} \cdot \mathbf{w} \mathrm{d} x$, $\forall \mathbf{w} \in H^{1}\left(\omega^{*} ; \mathbb{R}^{d}\right)$. Here we can appeal to the generalization of Weyl's theorem for elastic problems [17] and $\nu_{n+1}=\frac{4 \pi}{8\left(\sigma^{*}\right)^{2} / 3}(n+1)(1+o(1))$, for $d=2$ and $\nu_{n+1}=\left[6 \pi^{2} /(1+4 \sqrt{2})\left(\sigma^{*}\right)^{3} /\right]^{1 / 3}(n+1)^{2 / 3}(1+o(1))$ for $d=3$. The required upper bound on $T$ now follows and the theorem is proved.

Now we apply Lemma 7.6 together with Lemma 7.7 to obtain the following convergence rate associated with the family of approximation spaces $\mathcal{F}_{n}\left(\omega, \omega^{*}\right)$ given by

Theorem 7.8. Let $\mathbf{u} \in H_{\mathbb{A}}\left(\omega^{*} ; \mathbb{R}^{d}\right) / \mathcal{R}$ then there exists an approximation $\mathbf{v}_{u} \in \mathcal{F}_{n}\left(\omega, \omega^{*}\right)$ for which

$$
\left\|\mathbf{u}-\mathbf{v}_{u}\right\|_{\mathcal{E}(\omega)}=\inf _{\mathbf{v} \in \mathcal{F}_{n}\left(\omega, \omega^{*}\right)}\|\mathbf{u}-\mathbf{v}\|_{\mathcal{E}(\omega)} \leq I\left(\omega, \omega^{*}\right) C_{n}\|\mathbf{u}\|_{\mathcal{E}\left(\omega^{*}\right)}
$$

where

$$
I\left(\omega, \omega^{*}\right)=4 \theta_{d} \frac{1+\rho}{\rho}(\beta / \alpha)^{1 / 2} \text { and } C_{n}=n^{-1 / d}(1+o(1)), d=2,3 .
$$

Next we proceed iteratively to construct a family of local approximation spaces with a rate of convergence that is nearly exponential. For any pair of two concentric cubes $Q \subset Q^{*}$ we define $\mathcal{F}_{n}\left(Q, Q^{*}\right)$ to be the space given by the restriction of $W_{n}\left(Q^{*}\right)$ on $Q$. We suppose that $\omega^{*}$ is of side length $\sigma^{*}$. Let $N>1$ be an integer and we suppose that $\omega$ is of side length $\sigma$ and $\sigma^{*}=\sigma(1+\rho)$. Choose $\omega_{j}, j=1,2, \ldots, N$ to be the nested family of concentric cubes with side length $\sigma(1+\rho(N+1-j) / N)$ for which $\omega=\omega_{N+1} \subset \omega_{N} \subset \omega_{N-1} \subset \cdots \subset \omega_{1}=\omega^{*}$. We introduce the local spaces, $\mathcal{F}_{n}\left(\omega, \omega_{N}\right), \mathcal{F}_{n}\left(\omega, \omega_{N-1}\right), \ldots, \mathcal{F}_{n}\left(\omega, \omega_{1}\right)$. Put $m=N \times n$ and we define the approximation space given by

$$
\mathcal{T}\left(m, \omega, \omega^{*}\right)=\mathcal{F}_{n}\left(\omega, \omega_{1}\right) \oplus \cdots \oplus \mathcal{F}_{n}\left(\omega, \omega_{N}\right) .
$$

Theorem 7.9. Let $\mathbf{u} \in H_{\mathbb{A}}\left(\omega^{*}\right) / \mathcal{R}$ and $N$ be an integer such that $1 \leq N \leq n^{\gamma}$, with $0<\gamma<1 / d$. Then there exists $\mathbf{z}_{u} \in \mathcal{T}\left(m, \omega, \omega^{*}\right)$ such that

$$
\left\|\mathbf{u}-\mathbf{z}_{u}\right\|_{\mathcal{E}(\omega)} \leq \varsigma^{N}\|\mathbf{u}\|_{\mathcal{E}\left(\omega^{*}\right)}
$$

and $\varsigma=4 \theta_{d} \frac{1+\rho}{\rho} N(\beta / \alpha)^{1 / 2} C_{n}, \quad d=2,3$.

Theorem 7.9 and the associated exponential decay given by Theorem 7.3 now follow from identical arguments to those presented in [7]. The proof of Theorem 7.5 (exponential convergence for boundary domains) proceeds along the same lines as the proof of Theorem 7.3 given above. Here one applies the elastic analog of Lemma 3.4 of [7], together with the version of the Caccioppoli inequality for boundary domains developed in [8] and the asymptotic decay result of [17].

\section{Summary AND PERSPECTIVE}

Recent developments in aerospace and infrastructure are driving the need for new numerical methods for the recovery of local fields inside of large systems composed of heterogeneous structures. Several different approaches have been developed to address these problems as well as for complex multiscale problems in geology and biology. Even though it is possible to directly apply FEM methods to these problems the primary challenge facing a direct approach is that the linear system will be extremely large typically with degrees of freedom on the order $10^{9}$ and higher. This requires methods which are well suited to massively parallel computation. For heterogeneous media with high contrast and millions of interfaces between component materials the use of multigrid methods is problematic because of the high contrast and non-uniform meshes etc. The use of iterative 
methods require suitable preconditioners adapted to the problem at hand. Gaussian elimination methods based on $\mathcal{H}$-matrices [14] are not well parallelizable for three dimensional problems. There are also issues with the control of accuracy when using nonuniform meshes and higher order elements.

The implementation of the MS-GFEM method proposed here delivers the discrete solution operator using the same order of operations as the number of fibers inside the computational domain. This implementation is optimal in that the number of operations for solution is of the same order as the input data for the problem. The size of the MS-GFEM matrix used to represent the discrete inverse operator is controlled by the scale of the coarse grid and the convergence rate of the spectral basis and can be of order far less than the number of fibers. This strategy is general and can be applied to the solution of very large FE systems associated with the discrete solution of elliptic PDE.

The multiscale spectral GFEM proposed here can be viewed as a type of homogenization method with buffers (oversampling) i.e., with overlapping nonconforming elements glued together through a partition of unity. However there are other approaches to overlapping nonconforming elements such as the mortar method where the "overlap" is restricted to the boundaries of the "coarse" elements [1]. Here questions of convergence are delicate and solving the system of equations for the mortar method may be expensive.

For the implementation proposed here it is seen that the cash memory available to core processors places an upper limit on the size of the linear system used to construct the local approximation space. For currently available processors this limit is on the order $10^{6}$ degrees of freedom. The size of the local domain $\omega^{*}$ also influences the global computation. Here the size of $\omega^{*}$ influences the number of shape functions required for a given accuracy and hence the size $\mathrm{m}$ of the blocks in the matrix of the MS-GFEM and the operations required in the direct solve is $\mathcal{O}\left(\mathrm{m}^{3} \mathrm{H}^{-3}\right)$. Fortunately the condition number for the global solve appears weakly ldependent on $\mathrm{m}$ and is $\mathcal{O}\left(\mathrm{H}^{-2}\right)$ so that an iterative method with an inexpensive preconditioner could still be very effective.

We point out that in view of the estimates given in previous sections it is more or less clear that the approach using only a uniform mesh and the a priori convergence $\mathcal{O}\left(h^{1 / 2}\right)$ will be expensive for obtaining accuracy within the prescribed tolerance $\tau$. For the the circular fibers treated here we know that the solution and the local the eigenfunctions are piecewise analytic. For this case it is possible to utilize this and use "wiggly" domains $\omega^{*}$ to obtain an $\mathcal{O}(h)$ a priori convergence rate see, Remark 5.3. In the absence of a higher regularity theory for heterogeneous media one very likely needs to develop a-posteriori error indicators and estimators. The Hypothesis 4.1 on the constants appearing in the Besov space regularity inequality (4.34) is motivated by the analogous but rigorous estimates for the optimal basis for harmonic functions on the disk. The Hypothesis 5.1 is related to the question of separation of successive eigenvalues for elliptic eigenvalue problems [11]. The specific dependence of the separation between successive eigenvalues is delicate and remains an open question for future investigation.

The MS-GFEM proposed here has the advantage that the accuracy of the method is governed by the accuracy of the local shape functions and the dimension of the approximation space all of which are localized to a patch $\omega^{*}$. Hence we can adaptively choose the dimension of local approximation spaces and meshes inside each of the local domains $\omega^{*}$. Here we can use the Caccioppoli inequality to estimate the energy norm of the solution in $\omega^{*}$ and utilize this information within an error estimator. The MS-GFEM is very flexible and we can choose local domains $\omega^{*}$ of different sizes depending on the location within the computational domain $\Omega$. For example they can be of larger size in the middle of the domain $\Omega$ and can be smaller in size closer to the boundary $\partial \Omega$. Additionally the construction of conforming meshes which coincide with the boundaries of the fibers is possible although not inexpensive. See e.g. the meshes used in [3]. For fibers with circular cross section the GFEM/XFEM method together with SGFEM using the uniform mesh and enrichment (see e.g. $[5,55])$ has high potential for use in building local bases inside each $\omega^{*}$.

We conclude noting that we have addressed the implementation for a two dimensional problem to fix ideas. The MS-GFEM applies without modification to higher dimensional problems see, Section 7 where local spectral bases with exponential convergence are developed for general $L^{\infty}$ coefficients in two and three dimensions. This type of approach is also not only restricted to elliptic equations. 


\section{REFERENCES}

[1] T. Arbogast and K.J. Boyd, Subgrid upscaling and mixed multiscale finite elements. SIAM J. Numer. Anal. 44 (2006) $1150-1171$.

[2] I. Babuška, Homogenization and Its Application. Mathematical and Computational Problems. SYNSPADE 1975, Numer. Solution Part. Differ. Eqs. lll, edited by B. Hubbard. Academic Press (1976) 89-116.

[3] I. Babuška, B. Anderson, P. Smith and K. Levin, Damage analysis of fiber composites, Part I Statistical analysis on fiber scale. Comput. Methods Appl. Mech Engrg. 172 (1999) 27-77.

[4] I. Babuška, U. Banerjee and J. Osborn, Generalized Finite Element Methods-Main Ideas, Results and Perspective. Int. J. Comput. Methods 1 (2004) 67-103.

[5] I. Babuška and U. Banerjee, Stable Generalized Finiter Element Methods (SGFEM). Comput. Meth. Appl. Mech. Eng. 201204 (2012) 91-111.

[6] I. Babuška, G. Caloz and J.E. Osborn, Special finite element methods for a class of second order elliptic problems with rough coefficients. SIAM J. Numer. Anal. 31 (1994) 945-981.

[7] I. Babuška and R. Lipton, Optimal local approximation spaces for Generalized Finite Element Methods with application to multiscale problems. Multiscale Model. Simul., SIAM 9 (2011) 373-406.

[8] I. Babuška and R. Lipton, $L^{2}$ global to local projection: an approach to multiscale analysis. M3AS 21 (2011) $2211-2226$.

[9] I. Babuška and J. Melenk, The Partition of Unity Finite Element Method. Internat. J. Numer. Methods Engrg. 40 (1997) $727-758$.

[10] I. Babuška, J, E. Osborn, Generalized finite element methods:Their performance and their relation to the mixed methods. SIAM, J. Numer. Anal. 20 (1983) 510-536.

[11] I. Babuška and J.E. Osborn, Eigenvalue Problems. Handbook of Numerical Analysis, Finite Element Methods (Part 1), Vol. II, edited by P.G. Ciarlet and J.L. Lions. Elsevier Science Publishers, Amsterdam (1991).

[12] N.S. Bakhvalov and G. Panasenko, Homogenization Processes in Periodic Media. Nauka, Moscow (1984).

[13] R.M. Barrer, Diffusion and permeation in heterogenous media. Diffusion in Polymers, edited by J. Crank, G.S. Park. Academic Press (1968).

[14] M. Bebendorf and W. Hackbusch, Existence of $\mathcal{H}$-matrix approximants to the inverse FE-matrix of elliptic operators with $L^{\infty}$ coefficients. Numer. Math. 95 (2003) 1-28.

[15] L. Berlyand and H. Owhadi, Flux norm approach to finite dimensional homogenization approximations with nonseparated length scales and high contrast. Arch. Rat. Mech. Anal. 198 (2010) 177-221.

[16] A. Besounssan, J.L. Lions and G.C. Papanicolau, Asymptotic Analysis for Periodic Structures. North Holland Pub., Amsterdam (1978).

[17] T. Burchuladze and R. Rukhadze, Asymptotic distribution of eigenfunctions and eigenvalues of the basic boundary-contact oscillation problems of the classical theory of elasticity. Georgian Math. J. 6 (1999) 107-126.

[18] C.C. Chams and G.P. Sendeckij, Critique on theories predicting thermoelastic properties of fibrous composites. J. Comput. Mat. 2 (1968) 332-358.

[19] W. E, B. Engquist, The heterogeneous multiscale methods. Commun. Math. Sci. 1 (2003) 87-132.

[20] Weinan E, P. Ming and P. Zhang, Analysis of the heterogeneous multiscale method for elliptic homogenization problems. J. Amer. Math. Soc. 18 (2005) 121-156.

[21] Y. Efendiev and T.Y. Hou, Multiscale Finite Element Methods. Springer (2009).

[22] Y.R. Efendiev, T.Y. Hou and X.H. Wu, Convergence of a nonconforming mutiscale finite element method. SIAM J. Numer. Anal. 37 (2000) 888-910.

[23] Y. Efendiev and T. Hou, Multiscale finite element methods for porous media flows and their applications. Appl. Numer. Math. 57 (2007) 577-596.

[24] B. Engquist and P.E. Souganidis, Asymptotic and numerical homogenization. Acta Numer. 17 (2008) 147-190.

[25] J. Fish and Z. Huan, Multiscale enrichment based on partition unity. Int. J. Num. Mech. Eng. 62 (2005) $1341-1359$.

[26] S.K. Garg, V. Svalbonas and G.A. Gurtman, Analysis of Structural Composite Materials. Marcel Dekker, New York (1973).

[27] L. Grasedyck, I. Greff and S. Sauter, The AL basis for the solution of elliptic problems in heterogeneous media. Multiscale Model. Simul. 10 (2012) 245-258.

[28] L.J. Gurtman and R.H. Krock, Composite Materials, in vol II of Mechanics of composite materials, edited by G.P. Sendeckyj. Academic Press (1974).

[29] Z. Hashin, Theory of Fiber reinforced materials, NASA Report CR-1974 (1972) 1-704.

[30] T.Y. Hou and Xiao-Hui Wu, A multiscale finite element method for elliptic problems in composite materials and porous media. J. Comput. Phys. 134 (1997) 169-189.

[31] T.Y. Hou, Xiao-Hui Wu and Yu Zhang, Removing the cell resonance error in the multiscale finite element method via a Petrov-Galerkin formulation. Commun. Math. Sci. 2 (2004) 185-205.

[32] T.J.R. Hughes, Multiscale phenomena: Green's functions, the Dirichlet-to-Neumann formulaion, subgrid scale models, bubbles and the origins of stabilized methods. Comput. Methods Appl. Mech. Engrg. 127 (1995) 387-401.

[33] T.J.R. Hughes, G.R. Feijoo, L.Mazzei and J.B. Quincy, The variational multiscale method. A Paradigm for computational mechanics. Comput. Meth. Appl. Mech. Eng. 166 (1998) 3-24.

[34] K. Lichtenecker, Die Electrizitatskonstante naturlicher und kustlicher Mischkorper. Phys. Zeitschr. XXVII (1926) 115-158. 
[35] A. Malquist, Multiscale methods for elliptic problems. Multiscale Model. Simul. 9 (2011) 1064-1086.

[36] G.F. Masotti, Discussione analitica sul influenze che L'azione di mezo dialettrico hu sulla distribuziione dell' electtricita alla superficie di pin corpi ellecttici diseminati in esso. Mem.Di Math. et di Fisica in Modena 24 (1850) 49.

[37] G. Maxwell, Trestise on Electricity and Magnetisum, vol. 1. Oxford Univ. Press (1873) 62.

[38] J. Melenk and I. Babuška, The Partion of Unity Method Basic Theory and Applications, Comput. Meth. Appl. Mech. Eng. 139 (1996) 289-314.

[39] J.M. Melenk, On n-widths for elliptic problems. J. Math. Anal. Appl. 247 (2000) 272-289.

[40] G.W. Milton. The Theory of Composites. Cambridge University Press, Cambridge (2002).

[41] F. Murat, H-convergence, Séminaire d'Analyse Fonctionelle et Numérique de l'Université d'Alger, mimeographed notes, 1978. L. Tartar Cours Peccot, College de France (1977). Translated into English as F. Murat L. Tartar, H- convergence, in Topics in the Mathematical Modeling of Composite Materials, Progress in Nonlinear Differential Equations and their Applications, in vol. 31, edited by A.V. Cherkaev, R.V. Kohn. Birkhäuser, Boston (1997) 21-43.

[42] J. Nolen, G. Papanicolaou and O. Pironneau, A framework for adaptive multiscale methods for elliptic problems. Multiscale Model. Simul. 7 (2008) 171-196.

[43] H. Owhadi and L. Zhang, Metric-based upscaling. Commun. Pure Appl. Math. 60 (2007) 675-723.

[44] H. Owhadi and L. Zhang, Localized bases for finite-dimensional homogenization approximations with nonseparated scales and high contrast. Multiscale Model. Simul. 9 (2011) 1373-1398.

[45] A. Pinkus, $n$-Widths in Approximation Theory. Springer-Verlag, Berlin, Heidelberg, New York 7 (1985).

[46] S.D. Poisson, Second mem. sur la theorie de magnetism, Mem. de L Acad. de France (1822) 5.

[47] J.W. Rayleigh, On the influence of obstacles in rectangular order upon the properties of the medium. Philos. Mag. 50 (1892) 481.

[48] E. Sanchez-Palencia. Non-Homogeneous Media and Vibration Theory, in vol. 127 of Lecture Notes in Physics. Springer-Verlag (1980).

[49] S. Spagnolo, Sul limite delle soluzioni di problemi di Cauchy relativi all'equazione del calore. Ann Scu. Norm. Pisa 21 (1967) 657-699.

[50] S. Spagnolo, Sulla convergenza di soluzioni di equazioni paraboliche ed ellittiche. Ann. Sc. Norm. Sup. Pisa 22 (1968) 517-597.

[51] S. Spagnolo, Convergence in Energy for Elliptic Operators, edited by B. Hubbard. Numer. Solutions Partial Differ Eqs. III, (Synspade 1975, College Park, Maryland 1975). Academic Press, New York (1975).

[52] W. Streider and R. Aris, Variational Methods Applied to Problems of Diffusion and Reaction, Springer Tracts in Natural Philosophy. Springer-Verlag (1973).

[53] T. Strouboulis, L. Zhang and I Babuška, Generalized finite element method using mesh-based handbooks application to problem in domains with many voids. Comput. Methods Appl. Mechanics Engrg. 192 (2003) 3109-3161.

[54] T. Strouboulis, I. Babuška and K. Copps, The design and analysis of the generalized finite element method. Comput. Methods Appl. Mech. Engrg. 181 (2001) 43-69.

[55] T. Strouboulis, L. Zhang and I. Babuška, p-version of generalized FEM using mesh based handbooks with applications to multiscale problems. Int. J. Num. Meth. Engrg. 60 (2004) 1639-1672.

[56] S. Torquato, Random Heterogeneous Materials, Microstructure and Macroscopic Properties. Springer, New York (2002). 